\title{
Structure and dynamics of a mycobacterial type VII secretion system
}

https://doi.org/10.1038/s41586-021-03517-z

Received: 30 November 2020

Accepted: 6 April 2021

Published online: 12 May 2021

\section{Open access}

Check for updates

\author{
Catalin M. Bunduc ${ }^{1,2,3,4}$, Dirk Fahrenkamp ${ }^{1,2,3}$, Jiri Wald ${ }^{1,2,3}$, Roy Ummels ${ }^{5}$, Wilbert Bitter ${ }^{4,5}$, \\ Edith N. G. Houben ${ }^{4} \&$ Thomas C. Marlovits ${ }^{1,2,3 凶}$
}

Mycobacterium tuberculosis is the cause of one of the most important infectious diseases in humans, which leads to 1.4 million deaths every year ${ }^{1}$. Specialized protein transport systems-known as type VII secretion systems (T7SSs) - are central to the virulence of this pathogen, and are also crucial for nutrient and metabolite transport across the mycobacterial cell envelope ${ }^{2,3}$. Here we present the structure of an intact T7SS inner-membrane complex of M. tuberculosis. We show how the 2.32-MDa ESX-5 assembly, which contains 165 transmembrane helices, is restructured and stabilized as a trimer of dimers by the $\mathrm{MycP}_{5}$ protease. A trimer of $\mathrm{MycP}_{5}$ caps a central periplasmic dome-like chamber that is formed by three $\mathrm{EccB}_{5}$ dimers, with the proteolytic sites of $\mathrm{MycP}_{5}$ facing towards the cavity. This chamber suggests a central secretion and processing conduit. Complexes without $\mathrm{MycP}_{5}$ show disruption of the $\mathrm{EccB}_{5}$ periplasmic assembly and increased flexibility, which highlights the importance of $\mathrm{MycP}_{5}$ for complex integrity. Beneath the $\mathrm{EccB}_{5}-\mathrm{MycP}_{5}$ chamber, dimers of the $\mathrm{EccC}_{5}$ ATPase assemble into three bundles of four transmembrane helices each, which together seal the potential central secretion channel. Individual cytoplasmic $\mathrm{EccC}_{5}$ domains adopt two distinctive conformations that probably reflect different secretion states. Our work suggests a previously undescribed mechanism of protein transport and provides a structural scaffold to aid in the development of drugs against this major human pathogen.
Mycobacterium tuberculosis encodes five homologous, but functionally distinct, T7SSs that are designated ESX-1 to ESX-5. These systems translocate a number of effector proteins across the unique and impermeable diderm cell envelope ${ }^{2,3}$. Because of their importance for mycobacterial physiology and virulence, T7SSs are considered to be promising targets for the development of drugs for the treatment or prevention of tuberculosis ${ }^{4}$. Although T7SSs have previously been shown to form hexameric complexes ${ }^{5}$, high-resolution structural information exists only for part of the T7SS-a dimeric ESX-3 subcomplex from the nonpathogenic species Mycobacterium smegmatis ${ }^{6,7}$. Here we reconstituted the ESX-5 T7SS of M. tuberculosis H37Rv in M. smegmatis to obtain a structural view of the entire T7SS membrane complex from this human pathogen.

\section{Architecture and stoichiometry}

The M. tuberculosis ESX-5 system showed robust expression in M. smegmatis and correct assembly of the membrane complex (Extended Data Fig. 1a,b). Purification of the M. tuberculosis ESX-5 membrane complex (using a C-terminal Strep tag on $\mathrm{EccC}_{5}$ and mild solubilization conditions) resulted in copurification of the conserved $\mathrm{MycP}_{5}$ protease (Extended Data Fig.1c-e). To our knowledge, MycP (also known as myco$\sin )$ is absent in all previously reported T7SS structures ${ }^{5-7}$-although
MycP is known to be essential for T7SS function and complex stability ${ }^{8}$. The addition of nucleotides and $\mathrm{MgCl}_{2}$ improved sample homogeneity, as judged by a more-distinct high molecular weight complex on native-PAGE, size exclusion chromatography and subsequent negative-stain electron microscopy analysis (Extended Data Fig. $1 \mathrm{f}-\mathrm{j}$ ).

Cryo-electron microscopy (cryo-EM) analysis of the M. tuberculosis ESX-5 complex purified in the presence of $\mathrm{ADP}-\mathrm{AIF}_{3}$ showed clear hexameric particles (Extended Data Figs. 2b,3). We performed an ab initio reconstruction without symmetry enforcement that yielded an average resolution of approximately $4 \AA$ (Extended Data Fig. 4), which improved to an overall resolution of approximately $3.5 \AA$ after further data processing; this allowed us to build around $78 \%$ of the stable complex de novo (Supplementary Tables 1,2). The intact machinery comprises $\mathrm{EccB}_{5}, \mathrm{EccC}_{5}, \mathrm{EccD}_{5}, \mathrm{EccE}_{5}$ and $\mathrm{MycP}_{5}$ with a 6:6:12:6:3 stoichiometry (Fig. 1b, c, d, Supplementary Video 1) resulting in a 2.32-MDa complex that is anchored in the inner membrane through 165 transmembrane helices (TMHs) (Fig. 1e). The membrane assembly is best described as a trimer of dimers, in which each dimer comprises a single copy of $\mathrm{MycP}_{5}$ and two protomers each of one copy of $\mathrm{EccB}_{5}, \mathrm{EccC}_{5}, \mathrm{EccE}_{5}$ and two copies of $\mathrm{EcCD}_{5}$ (Fig. 1f). The overall fold and stoichiometry of a dimeric building block of $M$. tuberculosis ESX-5 is similar to that of a dimer of ESX-3 from M. smegmatis ${ }^{6,7}$, albeit with notable differences on the periplasmic side and the angle between protomers. In the intact

${ }^{1}$ Centre for Structural Systems Biology, Hamburg, Germany. ${ }^{2}$ Institute of Structural and Systems Biology, University Medical Centre Hamburg-Eppendorf, Hamburg, Germany. ${ }^{3}$ Deutsches Elektron Synchrotron DESY, Hamburg, Germany. ${ }^{4}$ Molecular Microbiology Section, Amsterdam Institute of Molecular and Life Sciences, Vrije Universiteit Amsterdam, Amsterdam, The Netherlands. ${ }^{5}$ Department of Medical Microbiology and Infection Control, Amsterdam Infection and Immunity Institute, Amsterdam UMC, Amsterdam, The Netherlands.

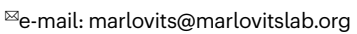




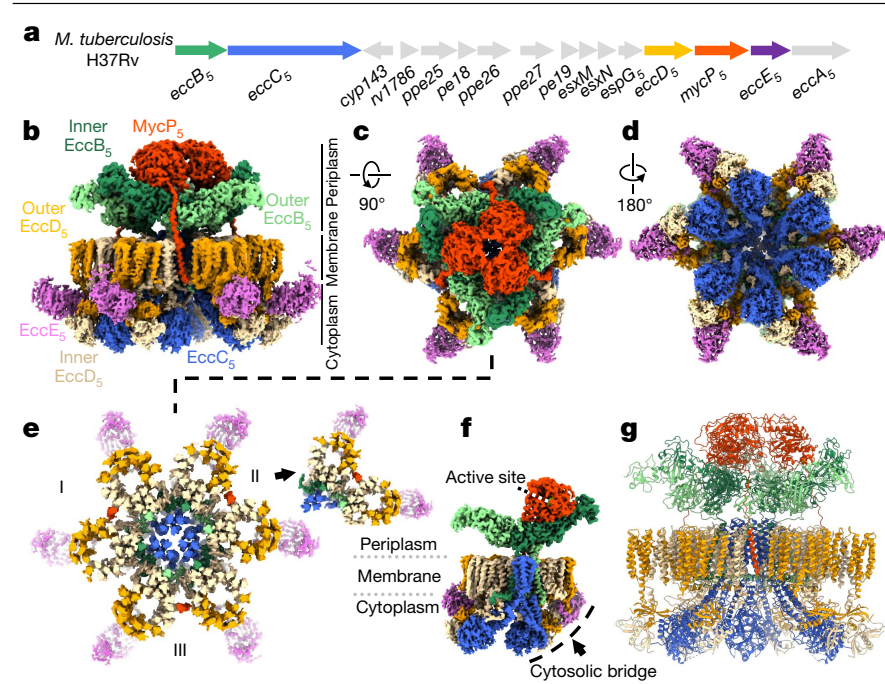

Fig. 1 Cryo-EM structure of the intact ESX-5 inner-membrane complex of M.tuberculosis. a, Genetic organization of the es $x$-5 locus of $M$. tuberculosis H37Rv, which was cloned and expressed in M. smegmatis $\mathrm{MC}^{2} 155$. b-e, Cryo-EM density of the intact ESX-5 inner-membrane complex of $M$. tuberculosis, zoned and coloured for every individual component. Components are inner $\mathrm{EccB}_{5}$ (darkgreen), outer $\mathrm{EccB}_{5}$ (light green), $\mathrm{EccC}_{5}$ (blue), inner $\mathrm{EcCD}_{5}$ (beige), outer $\mathrm{EccD}_{5}$ (orange), $\mathrm{EccE}_{5}$ (purple) and $\mathrm{MycP}_{5}$ (red). The full complex is $28.5 \mathrm{~nm}$ in width and $20 \mathrm{~nm}$ in height, and has an absolute stoichiometry of 6:6:12:6:3 for $\mathrm{EccB}_{5}: \mathrm{EccC}_{5}: \mathrm{EccD}_{5}: \mathrm{EccE}_{5}: \mathrm{MycP}_{5} \cdot \mathbf{b}-\mathbf{e}$, Side (b), top (c) and bottom (d) views and a top cross-section (e) of the complex at the membrane level, highlighting the arrangement of the 165-TMH region. Inset, top cross-section of an extracted dimeric unit.f, Single dimer viewed from the centre of the intact complex,

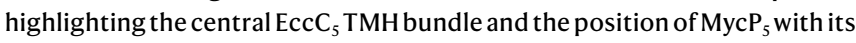
active site directed towards the inside of the periplasmic cavity. $g$, Ribbon model of the M. tuberculosis ESX-5 assembly.

ESX-5 complex, the angle between protomers at the membrane level differs by about $0.5^{\circ}$, from $59.7^{\circ}$ between protomers of one dimer to $60.2^{\circ}$ between protomers of adjacent dimers. However, at the cytosolic level these angles differ by more than $10^{\circ}$ (from $65.3^{\circ}$ to $54.7^{\circ}$ ) (Extended Data Fig. 5). By contrast, the individual ESX-3 dimer displayed an overall angle of $72^{\circ}$ between protomers ${ }^{6,7}$.

\section{Structural rearrangements of periplasmic domains}

The periplasmic assembly of the ESX-5 membrane complex of M.tuberculosis is formed by three $\mathrm{EccB}_{5}$ dimers and three $\mathrm{MycP}_{5}$ proteases. The $\mathrm{EccB}_{5}$ dimers assemble in a triangle, which forms a central cavity (Fig. 2c). Within an $\mathrm{EccB}_{5}$ dimer, two slightly different conformations (that is, inner and outer) can be distinguished between monomers, depending on their position. $\mathrm{EccB}_{5}$ dimerization is mediated mainly through the $\mathrm{R} 1$ and $\mathrm{R} 4$ repeat domains and is further stabilized by the $\mathrm{EccB}_{5} \mathrm{C}$ termini, which wrap around their interacting $\mathrm{EccB}_{5}$ partner to form intermolecular hydrophobic contacts with its R1 domain (Fig. 2d). The GIPGAP motif-which is a highly conserved region in EccB homologues-is central to these interactions (Fig. 2d). Compared to the $\mathrm{EccB}_{3}$ dimer from the ESX-3 subassembly ${ }^{6,7}$, the three $\mathrm{EccB}_{5}$ dimers are rotated by about $52^{\circ}$ with respect to their corresponding $\mathrm{EccC}_{5}-\mathrm{EccD}_{5}-\mathrm{EccE}_{5}$ membrane dimers, which indicates that large conformational rearrangements are required during maturation into the fully assembled hexamer (Extended Data Fig. 6d). To form the triangle-shaped assembly, the inner $\mathrm{EccB}_{5}$ engages the outer $\mathrm{EccB}_{5}$ of the adjacent dimer by packing its R3 domain against the $\alpha$-helices $\alpha 5$ and $\alpha 8$ of domains R2 and $\mathrm{R} 3$, respectively, which results in an asymmetric $\mathrm{EccB}_{5}$-tip arrangement (Fig. 2c). Consequently, domain R3 of the outer $\mathrm{EccB}_{5}$ does not form any interactions at its tip extremity and thus displays higher flexibility, consistent with previous observations ${ }^{9}$.

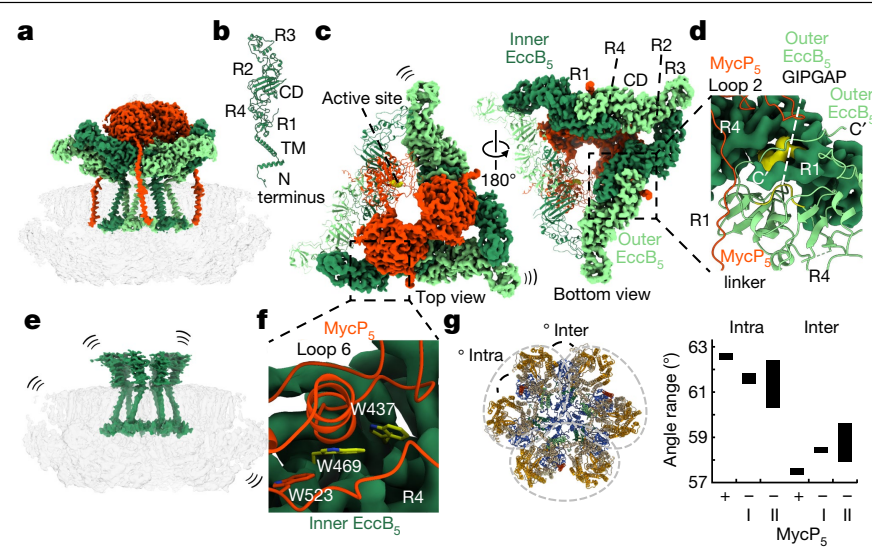

Fig. $2 \mid \mathrm{MycP}_{5}$ drives $\operatorname{EccB}_{5}$ hexamerization and stabilization of the membrane complex. a, Transparent assembly of intact $M$. tuberculosis ESX-5, with $\mathrm{EccB}_{5}$ and $\mathrm{MycP}_{5}$ coloured as in Fig. 1.b. Complete structure of monomeric M. tuberculosis $\mathrm{EccB}_{5}$, highlighting its overall fold and domains. c, Top and bottom view of the $\mathrm{EccB}_{5}-\mathrm{MycP}_{5}$ periplasmic assembly with one unit $\left(\mathrm{EccB}_{5}\right.$ dimer and $\mathrm{MycP}_{5}$ monomer) as ribbon model, highlighting the active site of $\mathrm{MycP}_{5}$ in yellow. $\mathrm{CD}$, central domain; $\mathrm{TM}, \mathrm{TMH}$. d, $\mathrm{EccB}_{5}$ dimerization site, highlighting the $\mathrm{C}$-terminus of outer $\mathrm{EccB}_{5}$ that is wrapped around the $\mathrm{R} 1$ and $\mathrm{R} 4$ domains of the adjacent inner $\mathrm{EccB}_{5}$ monomer, the conserved GIPGAP motif of $\mathrm{EccB}_{5}$ (in yellow) and the interactions of the $\mathrm{EccB}_{5}$ dimer with loop 2 and the linker connection of $\mathrm{MycP}_{5}$. e, Transparent map of $M$. tuberculosis ESX-5 without copurified $\mathrm{MycP}_{5}$, with $\mathrm{EccB}_{5}$ highlighted in dark green. The high flexibility of $\mathrm{EccB}_{5}$ and the overall heterogeneity of the membrane complex in the absence of $\mathrm{MycP}_{5}$ is indicated by curved lines. $\mathrm{f}, \mathrm{EccB}_{5}-\mathrm{MycP}_{5}$ interaction surface, highlighting the three buried tryptophans. $g$, Angle variation range between protomers of the $\mathrm{MycP}_{5}$-bound (+) and two unbound (-) states (I and II). Intra, between two protomers within a dimer: $62.7^{\circ}, 62.4^{\circ}$ and $62.4^{\circ}\left(\mathrm{MycP}_{5}\right.$-bound); $61.8^{\circ}, 61.3^{\circ}$ and $61.5^{\circ}$ (unbound, I); $61.4^{\circ}, 62.3^{\circ}$ and $60.2^{\circ}$ (unbound, II). Inter, between two protomers of adjacent dimers: $57.5^{\circ}, 57.3^{\circ}$ and $57.5^{\circ}$ ( $\mathrm{MycP}_{5}$-bound); $58.5^{\circ}, 58.3^{\circ}$ and $58.3^{\circ}$ (unbound, I); and $57.9^{\circ}, 58.3^{\circ}$ and $59.6^{\circ}$ (unbound, II).

\section{Periplasmic $\mathrm{MycP}_{5}-\mathrm{EccB}_{5}$ assembly}

Our periplasmic M. tuberculosis ESX-5 map shows three $\mathrm{MycP}_{5}$ proteases that form a dome-like structure, which cap the periplasmic central cavity (Fig. 2a, c, Supplementary Video 2). Interactions between $\mathrm{EccB}_{5}$ and $\mathrm{Myc}_{5}$ are mediated mainly by the $\mathrm{Myc}_{5}$ protease domain and a composite interface that is generated by the R4 domain and loop 6 (residues Thr424 to Ser435) of the inner $\mathrm{EccB}_{5}$ (Fig. 2f). The $\mathrm{MycP}_{5}-$ $\mathrm{EccB}_{5}$ interface covers a surface area of about $1,230 \AA^{2}$, which leads to the burial of three conserved tryptophan residues (Trp437 and Trp469 of $\mathrm{EccB}_{5}$, and Trp523 of $\mathrm{MycP}_{5}$ ) (Fig. 2f). Additionally, loop 2 of $\mathrm{MycP}_{5}$ binds to the $C$ terminus of the outer $\mathrm{EccB}_{5}$, which explains why a deletion of this loop previously showed reduced ESX-5 secretion in $\mathrm{Myco}$ bacterium marinum ${ }^{10}$ (Fig. 2d, Extended Data Fig. 6i). $\mathrm{MycP}_{5}-\mathrm{MycP}_{5}$ interactions are mediated mainly through loop 1 and the $\mathrm{N}$-terminal extension (which run across the top of the $\mathrm{MycP}_{5}$ protomers), and loop 3, which contacts the neighbouring protease domain from the side (Extended Data Fig. 7c, d). Loop 5 (residues Ala151 to Val271), which is cleaved during ESX-5 maturation ${ }^{11}$, folds along the interface of two protease domains towards the central pore formed by the $\mathrm{MycP}_{5}$ trimer (Extended Data Fig. 7a). Although we could not build a complete model of loop 5 (owing to its high flexibility), this loop appears to cap the central periplasmic pore (Extended Data Fig. 7a). Notably, as loop 5 is not present in all mycosins and is dispensable for ESX-5 secretion ${ }^{10}$, a speculative role in gating remains to be identified. The active sites of the $\mathrm{MycP}_{5}$ proteases face towards the central lumen of the cavity (Fig. 2c), which implies that potential substrates of this protease are translocated through-and processed within-this periplasmic chamber.

The dimer interface between the inner and outer $\mathrm{EccB}_{5}$ is the largest in the periplasmic assembly, and covers a surface area of around 
$2,000 \AA^{2}$ and provides a solvation-free energy gain of $\Delta G=-23 \mathrm{kcal} \mathrm{mol}^{-1}$ per dimer (Fig. 2d). By contrast, the interfaces formed between $\mathrm{EccB}_{5}$ dimers each bury a surface area of about $600 \AA^{2}$, with a cumulative energy gain of only $\Delta G=-18 \mathrm{kcal} \mathrm{mol}^{-1}$ upon trimerization. This could provide an explanation as to why dimeric ESX subcomplexes are more stable than their fully assembled counterparts ${ }^{6,7}$. The intermolecular $\mathrm{EccB}_{5}-\mathrm{MycP}_{5}$ interactions (which have a surface area of around $395 \AA^{2}$ and $\left.\Delta G=-0.1 \mathrm{kcal} \mathrm{mol}^{-1}\right)$ are even more modest, which provides a rationale for why interactions between $\mathrm{MycP}_{5}$ and the membrane complex have so far remained unknown.

\section{$\mathbf{M y c P}_{5}$ stabilizes the entire membrane complex}

To further investigate the effect of $\mathrm{MycP}_{5}$ on the entire structure, we analysed $\mathrm{MycP}_{5}$-free $M$. tuberculosis $\mathrm{ESX}-5$ complexes from the same preparation (Fig. 2e, g). These assemblies contained the same $\mathrm{EccB}_{5}: \mathrm{EccC}_{5}: \mathrm{EccD}_{5}: \mathrm{EccE}_{5}$ stoichiometry as the fully assembled complexes (Extended Data Fig. 8). Following 3D reconstruction, we obtained two $\mathrm{MycP}_{5}$-free $M$. tuberculosis ESX-5 maps that displayed resolution estimates of about 4.5 and about $6.7 \AA$ (Extended Data Fig. 4). The differences were most notable on the periplasmic side, on which the six $\mathrm{EccB}_{5}$ copies showed high flexibility and did not form a stable triangular scaffold in the absence of $\mathrm{MycP}_{5}$ (Fig. 2e). This shows that $\mathrm{MycP}_{5}$ enables the trimerization of the $\mathrm{EccB}_{5}$ dimers in the periplasm. This result is highly interesting, because mycosins are subtilisin-like proteases without any additional domains apart from a TMH, some small loops and an $\mathrm{N}$-terminal extension that wraps around the protein ${ }^{12,13}$. A more structural role for mycosin has previously been predicted ${ }^{8}$ but we now understand the essential role of mycosins in T7SSs.

In contrast to the periplasmic domain, the cytosolic and membrane regions of the $\mathrm{MycP}_{5}$-free maps were more similar to those of the $\mathrm{MycP}_{5}$-containing particles (Extended Data Fig. 8). However, the $\mathrm{MycP}_{5}$-free particles displayed an increased heterogeneity that affected the entire complex, which resulted in a slight waving of the membrane region and an increased angle variation between individual protomers (Fig. 2g, Extended Data Fig. 8, Supplementary Video 4). The protease domain and $\mathrm{TMH}$ of $\mathrm{MycP}_{5}$ synergistically reinforce the membrane complex. Their interactions with periplasmic inner $\mathrm{EccB}_{5}$ and membrane-embedded outer $\mathrm{EccD}_{5}$ (from separate protomers within a dimer) better anchor the periplasmic assembly to the membrane, while also stabilizing the dimeric unit (Extended Data Figs. 8f, 9). Additionally, by driving the formation of the periplasmic assembly, $\mathrm{MycP}_{5}$ stabilizes the entire complex by promoting cross-dimer $\mathrm{MycP}_{5}-\mathrm{MycP}_{5}$ and inner $\mathrm{EccB}_{5}$-outer $\mathrm{EccB}_{5}$ interactions (Extended Data Fig. 9). Our $\mathrm{MycP}_{5}$-free $M$.tuberculosis ESX-5 maps are reminiscent of the hexameric, low-resolution negative-stain structure of ESX-5 from Mycobacterium xenopi, in which the periplasm was similarly disorganized in the absence of $\mathrm{MycP}_{5}{ }^{5}$.

\section{$\mathrm{EccC}_{5}$ gates a potential secretion conduit}

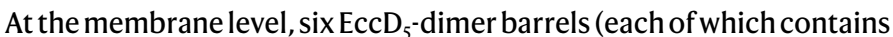
$22 \mathrm{TMHs}$ ) together form a circular raft with an inner cavity (Extended Data Fig. 10). Within this raft, inner $\mathrm{EcCD}_{5}$ monomers are situated closer to the centre, whereas outer $\mathrm{EccD}_{5}$ monomers face towards the periphery of the membrane complex. The $\mathrm{EccD}_{5}$ membrane barrels are structurally highly similar to the homologous $\mathrm{EccD}_{3}$ barrel in the ESX-3 subassembly ${ }^{6,7}$. The inner surface of each $\mathrm{EccD}_{5}$ barrel is decorated with densities that are attributable to stably bound lipids or detergent molecules, which suggests that, in their native membrane environment, these barrels are filled with membrane lipids (Extended Data Fig.10e, f).

The TMH of each copy of $\mathrm{EccB}_{5}$ is anchored within the confinement of the $\mathrm{EcCD}_{5}$ raft through hydrophobic interfaces that are provided by TMH6 and TMH11 of inner $\mathrm{EcCD}_{5}$ and stably bound lipids (Fig. 3a, Extended Data Fig. 6f). Notably, no substantial intermolecular
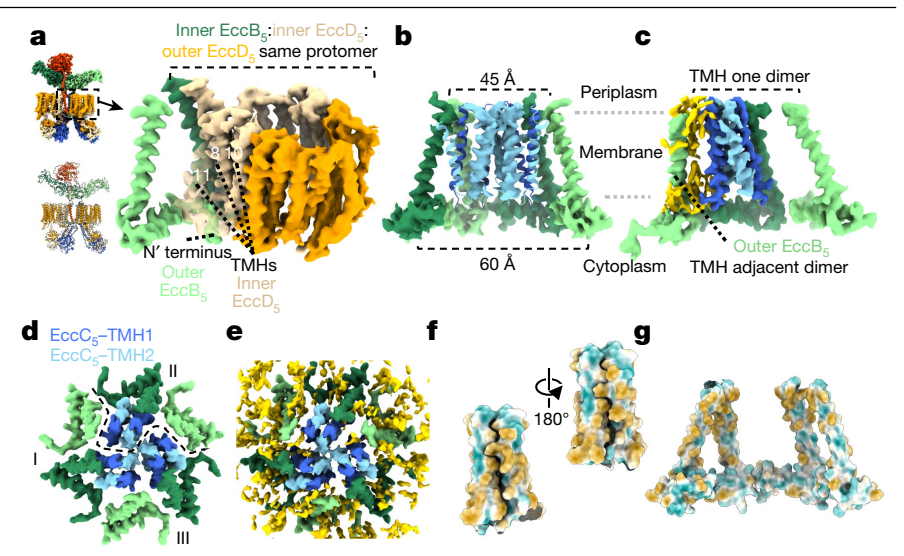

Fig. 3 | A basket formed by the EccB $_{5}$ TMHs holds three four-TMH bundles of $\mathbf{E c c C}_{5} \cdot \mathbf{a}$, Angled view from the outside of the complex, showing the TMH and $\mathrm{N}$ terminus of an outer $\mathrm{EccB}_{5}$ interacting with a pocket formed by TMH8, TMH10 and TMH11 of inner $\mathrm{EccD}_{5}$ from the adjacent barrel.b, Side cross-section through the $\mathrm{EccB}_{5}$ basket that contains the $\mathrm{EccC}_{5} \mathrm{TMH}$ bundles. Light blue densities depict the three copies of $\mathrm{EccC}_{5} \mathrm{TMH}_{2}$ that form the central pyramid. Two TMHs of $\mathrm{EccC}_{5}$ were removed for clarity. Sizes indicate the inner diameters of the $\mathrm{EccB}_{5}$ basket. c, Side cross-section through an $\mathrm{EccB}_{5}$ basket, showing that the $\mathrm{EccC}_{5} \mathrm{TMH}_{\mathrm{H}}$ bundle does not interact with outer $\mathrm{EccB}_{5}$ from its own dimer, but instead forms lipid-mediated interactions with the outer $\mathrm{EccB}_{5} \mathrm{TMH}_{\mathrm{T}}$ of the adjacent dimer. Lipids are shown in gold. $\mathbf{d}$, Top view of the central $\mathrm{EccB}_{5}$ basket and the $\mathrm{EccC}_{5} \mathrm{TMH}$ bundles. Dashed line marks the TMHs that belong to one dimeric unit.e, As in d, highlighting the lipid-rich environment. In the central area that surrounds the $\mathrm{EccC}_{5}$ pyramid, lipids are not clearly distinguishable (which suggests fluidity in this area).f, g, Surface model displaying the hydrophobicity of an $\mathrm{EccC}_{5} \mathrm{TMH}$ bundle $(\mathbf{f})$ and the $\mathrm{EccB}_{5}$ basket $(\mathbf{g})$. Hydrophilic amino acids are shown in turquoise, and hydrophobic residues are shown in sepia.

interactions can be found between adjacent $\mathrm{EccD}_{5}$ barrels. Instead, coupling between two neighbouring $\mathrm{EccD}_{5}$ barrels is achieved by the $\mathrm{N}$-terminal loop and $\alpha$-helix of $\mathrm{EccB}_{5}$ that run parallel to the cytoplasmic side of the inner membrane and engage in interactions with the TMHs of the neighbouring $\mathrm{EccD}_{5}$ barrel in a clockwise manner (Fig. 3a, Extended Data Fig. 6g, h). Because the TMH of $\mathrm{EccB}_{5}$ is slightly angled towards the centre of the complex, the architecture of the hexamer of $\mathrm{EccB}_{5}$ TMHs is reminiscent of a basket, the inner diameter of which shrinks from around $60 \AA$ to around $45 \AA$ towards the periplasmic side (Fig. $3 \mathrm{~b}$ ).

EccC is the only component that is present in all T7SSs (including in related systems in Firmicutes ${ }^{2}$ ), and is therefore thought to be the central component in this nanomachinery. Each EccC protein has-in addition to two TMHs-four FtsK/SpollIE-like ATPase domains (also known as nucleotide-binding domains (NBDs)) that are known to be important for secretion ${ }^{6,7,14}$. We fully resolved the twelve $\mathrm{EccC}_{5} \mathrm{TMHs}$ in the intact M. tuberculosis ESX-5 complex; these form three four-TMH bundles, each of which belongs to the $\mathrm{EccC}_{5}$ molecules of one dimer (Fig. 3b-d, Extended Data Fig. 11). These bundles are held together by hydrophobic interactions and effectively seal the central space of the membrane complex, which is enclosed by the $\mathrm{EccB}_{5}$ basket (Fig. 3d, Supplementary Video 3). Two $\mathrm{EccC}_{5} \mathrm{TMH}_{\mathrm{H}}$ from each bundle contact the TMH of the inner $\mathrm{EccB}_{5}$, which leaves the outer $\mathrm{EccB}_{5} \mathrm{TMH}$ unbound by $\mathrm{EccC}_{5}$ (Fig. 3c, d). At the very centre of the complex, one TMH of each bundle contributes to the formation of a pyramidal assembly that aligns with the periplasmic chamber (Extended Data Fig. 11d).

The chamber within the $\mathrm{EccB}_{5}$ basket appears to be filled with lipids. However, the density for these lipids is more ambiguous than that of the lipids in and around the $\mathrm{EcCD}_{5}$ barrels, which suggests that the lipids within this chamber are more fluid (Fig. 3e). Notably, the local resolution gradually increases when moving from the centre to the $\mathrm{EccB}_{5}$ basket, where the resolution is highest (Extended Data Fig. 11c). 


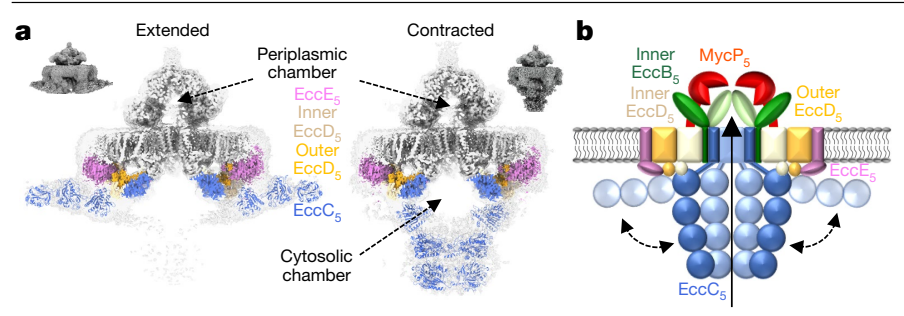

Fig. $4 \mid$ EccC $_{5}$ adopts an extended and a contracted conformation. a, Side cross-section of density maps, showing the extended and contracted conformation of $\mathrm{EccC}_{5}$. The periplasmic and cytoplasmic chambers formed by $\mathrm{EccB}_{5}-\mathrm{MycP}_{5}$ and by $\mathrm{EccC}_{5}$ upon closing are highlighted. Homology models of the three C-terminal NBDs of $\mathrm{EccC}_{5}$ are fitted in the cytosolic densities. Cytosolic bridge components are coloured as in Fig. 1.b, Model of the intact T7SS inner-membrane complex, highlighting the two conformations of $\mathrm{EccC}_{5}$.

This indicates that the $\mathrm{EccC}_{5} \mathrm{TMH}_{\text {bundles display more flexibility, as }}$ compared to the rigid $\mathrm{EccB}_{5}$ basket. The entrance to the putative $\mathrm{EccC}_{5}$ pore widens on the cytoplasmic side, where the $\mathrm{EccC}_{5}$ stalk domains expand radially (Extended Data Figs. 5,11a). Together, our data suggest that the six $\mathrm{EcCD}_{5}$ barrels provide a stable scaffold for assembly of a secretion pore that is confined by the $\mathrm{EccB}_{5} \mathrm{TMH}_{\mathrm{H}}$ and gated through

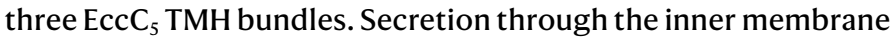
complex would require rearrangement of the $\mathrm{EccC}_{5}$ TMHs. Such a proposed central pore would extend into the periplasmic chamber that is formed by $\mathrm{EccB}_{5}$ and $\mathrm{MycP}_{5}$.

\section{Cytosolic $\mathrm{EccC}_{5}$ adopts two conformations}

At the cytoplasmic side of the complex, $\mathrm{EccC}_{5}$ has a stalk helix that connects its second TMH to the first NBD (which is also known as the DUF domain). This NBD is bound to the cytosolic domains of inner and outer $\mathrm{EccD}_{5}$, which-in turn-are bound to $\mathrm{EccE}_{5}$ at the periphery, together forming a 'cytosolic bridge' (Fig. 1f).

The distal C-terminal part of $\mathrm{EcCC}_{5}$, which comprises a string of three NBDs (NBD1, NBD2 and NBD3), adopts two main conformations: we refer to these as extended and contracted (Fig. $4 \mathrm{a})$. In the extended state, the C-terminal three $\mathrm{NBDs}$ of $\mathrm{EccC}_{5}$ bend parallel to the membrane, and align with the cytosolic domains of inner $\mathrm{EcCD}_{5}$ and $\mathrm{EccE}_{5}$ of the same protomer, and extend beyond the diameter of the membrane assembly. Although of considerably lower resolution, this density can confidently accommodate a homology model that consists of the three EccC ${ }_{5}$ NBD domains (Fig. 4a, Extended Data Fig. 12). Further classification of the extended state reveals $\mathrm{EccC}_{5}$ to be more heterogenous beyond NBD1, which suggests that NBD1 is more stably bound to components of its own protomer. Although we found only a relatively small number of particles in the contracted conformation, we solved the stable core of the membrane complex to sub-nanometre resolution (Extended Data Fig. 3). In the contracted state, the flexible arms of $\mathrm{EccC}_{5}$ extend from the interface between the DUF domain of $\mathrm{EccC}_{5}$ and the cytosolic domain of inner $\mathrm{EccD}_{5}$ (Fig. 4a, Extended Data Fig.12). We observed three separate disc-like structures that gradually constrict from the top to the bottom. This density shows a gap at the interface between NBD1 and NBD2. This would allow the previously postulated ${ }^{14,15}$ binding of substrates to the linker 2 that connects NBD1 and NBD2, resulting in the displacement of this linker and the activation of NBD1. The highly dynamic cytoplasmic domains of the machinery may provide the basis for substrate selection, recognition or transport across the membrane.

Our work provides a fully assembled structure of the ESX-5 inner-membrane complex of $M$. tuberculosis. As the membrane components of the five mycobacterial ESX systems show high sequence conservation, our results probably constitute a general structural blueprint for all of these T7SSs-including the virulence-related ESX-1 system (Supplementary Figs. 2, 3). Furthermore, our structure will serve as a platform for the identification of interactions that-if perturbed by small molecules-would aid in the treatment of tuberculosis.

\section{Online content}

Any methods, additional references, Nature Research reporting summaries, source data, extended data, supplementary information, acknowledgements, peer review information; details of author contributions and competing interests; and statements of data and code availability are available at https://doi.org/10.1038/s41586-021-03517-z.

WHO. Global Tuberculosis Report (WHO, 2020).

2. Bunduc, C. M., Bitter, W. \& Houben, E. N. G. Structure and function of the mycobacterial type VII secretion systems. Annu. Rev. Microbiol. 74, 315-335 (2020).

3. Gröschel, M. I., Sayes, F., Simeone, R., Majlessi, L. \& Brosch, R. ESX secretion systems: mycobacterial evolution to counter host immunity. Nat. Rev. Microbiol. 14, 677-691 (2016).

4. Rybniker, J. et al. Anticytolytic screen identifies inhibitors of mycobacterial virulence protein secretion. Cell Host Microbe 16, 538-548 (2014).

5. Beckham, K. S. et al. Structure of the mycobacterial ESX-5 type VII secretion system membrane complex by single-particle analysis. Nat. Microbiol. 2, 17047 (2017).

6. Famelis, N. et al. Architecture of the mycobacterial type VII secretion system. Nature 576, 321-325 (2019).

7. Poweleit, N. et al. The structure of the endogenous ESX-3 secretion system. eLife 8 , e52983 (2019).

8. van Winden, V. J. C. et al. Mycosins are required for the stabilization of the ESX-1 and ESX-5 type VII secretion membrane complexes. MBio 7, e01471-16 (2016).

9. Xie, X. Q. et al. Crystallographic observation of the movement of the membrane-distal domain of the T7SS core component EccB, from Mycobacterium tuberculosis. Acta Crystallogr. F 72, 139-144 (2016).

10. van Winden, V. J. C., Damen, M. P. M., Ummels, R., Bitter, W. \& Houben, E. N. G. Protease domain and transmembrane domain of the type VII secretion mycosin protease determine system-specific functioning in mycobacteria. J. Biol. Chem. 294, 4806-4814 (2019).

11. van Winden, V. J. C., Bunduc, C. M., Ummels, R., Bitter, W. \& Houben, E. N. G. A chimeric EccB-MycP fusion protein is functional and a stable component of the ESX-5 type VII secretion system membrane complex. J. Mol. Biol. 432, 1265-1278 (2020).

12. Solomonson, M. et al. Structure of the mycosin-1 protease from the mycobacterial ESX-1 protein type VII secretion system. J. Biol. Chem. 288, 17782-17790 (2013).

13. Wagner, J. M. et al. Understanding specificity of the mycosin proteases in ESX/type VII secretion by structural and functional analysis. J. Struct. Biol. 184, 115-128 (2013).

14. Rosenberg, O. S. et al. Substrates control multimerization and activation of the multi-domain ATPase motor of type VII secretion. Cell 161, 501-512 (2015).

15. Bunduc, C. M., Ummels, R., Bitter, W. \& Houben, E. N. G. Species-specific secretion of ESX5 type VII substrates is determined by the linker 2 of $\mathrm{EccC}_{5}$. Mol. Microbiol. 114, 66-76 (2020).

Publisher's note Springer Nature remains neutral with regard to jurisdictional claims in published maps and institutional affiliations.

Open Access This article is licensed under a Creative Commons Attribution 4.0 International License, which permits use, sharing, adaptation, distribution and reproduction in any medium or format, as long as you give appropriate credit to the original author(s) and the source, provide a link to the Creative Commons license, and indicate if changes were made. The images or other third party material in this article are included in the article's Creative Commons license, unless indicated otherwise in a credit line to the material. If material is not included in the article's Creative Commons license and your intended use is not permitted by statutory regulation or exceeds the permitted use, you will need to obtain permission directly from the copyright holder. To view a copy of this license, visit http://creativecommons.org/licenses/by/4.0/.

(c) The Author(s) 2021 


\section{Methods}

No statistical methods were used to predetermine sample size. The experiments were not randomized, and investigators were not blinded to allocation during experiments and outcome assessment.

\section{Molecular biology}

Escherichia coli Dh5 $\alpha$ was grown at $37^{\circ} \mathrm{C}$ and $200 \mathrm{rpm}$ in LB medium supplemented with $30 \mu \mathrm{g} \mathrm{ml}^{-1}$ streptomycin. Cloning was performed in $E$. coli $\mathrm{Dh} 5 \alpha$ using IProof DNA polymerase from BioRad and restriction enzymes from New England Biolabs. A list of the primers used for amplification is available in Supplementary Table 3.

The plasmid expressing $M$. tuberculosis ESX-5 was built as follows: the backbone of the previously described pMVESX- $5_{\text {mxen }}$ plasmid ${ }^{5}$ was modified to encode the unique restriction sites Dral and Pacl upstream and Spel and Ndel downstream of the TwinStrep tag sequence. The $r v 1782-r v 1783\left(e c c B_{5}-e c c C_{5}\right)$ region, including about 380 bp upstream of eccB $B_{5}$, of M. tuberculosis $\mathrm{H} 37 \mathrm{Rv}$ was amplified while adding Dral and Pacl restriction sites at the $5^{\prime}$ and $3^{\prime}$ ends, respectively (primers 1 and 2), and cloned into the modified plasmid upstream of the TwinStrep tag sequence, resulting in plasmid intermediate 1 . The $M$. tuberculosis H37Rv region spanning $r v 1791-r v 1798\left(\right.$ pe19-ecc $A_{5}$ ) was amplified while adding Spel and Ndel unique restriction sites (primers 3 and 4 ) and cloned downstream of the TwinStrep tag sequence into the intermediate 1 , resulting in plasmid intermediate 2 . Plasmid intermediate 2 was digested with Spel and SnaBI, removing the region rv1791-rv1794 ( $p e 19-e s p G_{5}$ ), and the region encompassing rv1785-rv1794 (cyp143esp $G_{5}$ ) was amplified as two individual PCR products (primers 5 and 6 and primers 7 and 8 ). The restricted backbone and PCR products were InFusion (Takara Bio)-ligated, resulting in the final pMV-ESX-5 $5_{\mathrm{mtb}}$ containing the entire $r v 1782-r v 1798\left(e c c B_{5}-e c c A_{5}\right)$ locus.

\section{Isolation of mycobacterial cell envelopes}

Mycobacterium smegmatis $\mathrm{MC}^{2} 155$ expressing M. tuberculosis ESX-5 was grown at $37^{\circ} \mathrm{C}$ and $90 \mathrm{rpm}$ in LB medium supplemented with $0.05 \%$ Tween 80 and $30 \mathrm{\mu g} \mathrm{ml}^{-1}$ streptomycin. Cultures were grown to an optical density (OD) at $600 \mathrm{~nm}$ of about 1.5 , spun down for $15 \mathrm{~min}$ at $12,000 \mathrm{~g}$ in a JLA-8.1000 rotor and subsequently washed in PBS. After culture collecting, all subsequent steps were performed at $4{ }^{\circ} \mathrm{C}$. Washed cell pellets were resuspended in buffer A $(50 \mathrm{mM}$ Tris- $\mathrm{HCl} \mathrm{pH} \mathrm{8,300} \mathrm{mM}$ $\mathrm{NaCl}$ and $10 \%$ glycerol) at a concentration of about $50 \mathrm{OD} \mathrm{ml}^{-1}$ and lysed by passing two times through a high-pressure homogenizer (Stansted) using a pressure of $0.83 \mathrm{kbar}$. Unbroken cells were pelleted at 5,000g for $5 \mathrm{~min}$ and supernatants were transferred to ultracentrifugation tubes. Cell envelopes were separated from the soluble fraction by ultracentrifugation at $150,000 \mathrm{~g}$ for $1.5 \mathrm{~h}$. Following ultracentrifugation, supernatants were discarded, pellets were washed once with buffer $A$, resuspended in buffer $A$ at a concentration of $750-1,000 \mathrm{OD} \mathrm{ml}^{-1}$, snap-frozen in liquid nitrogen and stored at $-80^{\circ} \mathrm{C}$ until further use. The protein concentration of the cell envelope fraction was measured by BCA assay (Pierce).

\section{Purification of the M. tuberculosis ESX-5 membrane complex}

All steps were performed at $4{ }^{\circ} \mathrm{C}$. The M. tuberculosis ESX-5 was purified as follows: cell envelope fractions were diluted to $3 \mathrm{mg} \mathrm{ml}^{-1}$ in buffer B (50 mM Tris- $\mathrm{HCl} \mathrm{pH} \mathrm{8,300} \mathrm{mM} \mathrm{NaCl}$ and 5\% glycerol), supplemented with $0.25 \% \mathrm{DDM}, 3 \mathrm{mM} \mathrm{ADP}-\mathrm{AlF}_{3}$ and $6 \mathrm{mM} \mathrm{MgCl}_{2}$. Following solubilization, the cell envelope mixture was spun down at $100,000 \mathrm{~g}$ for $20 \mathrm{~min}$, supernatants were collected and incubated with StrepTactin resin (IBA). Beads were subsequently washed with buffer $B$ supplemented with $0.03 \% \mathrm{DDM}, 1 \mathrm{mM} \mathrm{ADP}-\mathrm{AlF}_{3}$ and $2 \mathrm{mM} \mathrm{MgCl}_{2}$. Bound protein was eluted from the resin with buffer $B$ supplemented with $0.03 \% \mathrm{DDM}, 3 \mathrm{mM}$ ADP$\mathrm{AlF}_{3}, 6 \mathrm{mM} \mathrm{MgCl}_{2}$ and $10 \mathrm{mM}$ desthiobiotin. The protein concentration of the eluate was measured by Bradford assay and amphipol A8-35 was added in an amphipol:protein ratio of 5:1. After a 1-h incubation, the amphipol-containing eluate was incubated overnight (around 12-16 h) with BioBeads in a BioBeads:detergent ratio of 20:1. Subsequently, BioBeads were removed using gravity flow chromatography columns and the sample was concentrated using Amicon Ultra 0.5-ml 100-kDa spin concentrators. The concentrated sample was further purified through size exclusion chromatography (SEC), using a Superose 6 Increase column running in buffer $\mathrm{C}(20 \mathrm{mM}$ Tris- $\mathrm{HCl} \mathrm{pH} 8,200 \mathrm{mM}$ $\mathrm{NaCl}$ ) supplemented with $1 \mathrm{mM} \mathrm{ADP}-\mathrm{AlF}_{3}$ and $2 \mathrm{mM} \mathrm{MgCl}_{2}$. Size exclusion chromatography fractions were analysed by blue-native polyacrylamide gel electrophoresis (BN-PAGE) and negative-stain electron microscopy, after which the appropriate fractions were concentrated for cryo-EM using Amicon Ultra 0.5-ml 100-kDa spin concentrators. The initial Arctica sample was purified similarly, with the addition of $5 \%$ glycerol in the SEC purification step and the omission of $\mathrm{ADP}-\mathrm{AlF}_{3}$ and $\mathrm{MgCl}_{2}$ from the purification protocol.

\section{BN-PAGE}

Samples consisting of either solubilized membranes or purified membrane complexes were mixed with 5\% G-250 sample additive (Invitrogen), to a final concentration of about $0.2 \%$, and run on $3-12 \%$ NativePage Bis-Tris Protein Gels (Invitrogen) according to manufacturer specifications. Gels were either stained with Coomasie R-250 or transferred to PVDF membranes and stained with appropriate antibodies (Supplementary Fig. 1). Antisera against $\mathrm{EccB}_{5}$, used in Extended Data Fig. 1b, was raised against the synthetic peptide CLPMDMSPAELVVPK and has previously been described ${ }^{16}$. Polyclonal rabbit antisera against the peptide was raised in rabbits by Innovagen using Stimune (Prionix) as adjuvants. The antibody was used as a 1:5,000 dilution. Blots were visualized on a ChemoStar TouchMotionCor2 using ChemoStarTS.

\section{Negative-stain electron microscopy}

Carbon-coated copper grids were glow-discharged for $30 \mathrm{~s}$ at $25 \mathrm{~mA}$ using a GloQube Plus Glow Discharge System (Electron Microscopy Sciences). Four microlitres of diluted sample was applied to the grids and incubated for $30 \mathrm{~s}$. The sample was blotted off from the side and the grid was washed briefly with $4 \mu$ l of staining solution ( $2 \%$ uranyl acetate) and then stained with $4 \mu \mathrm{l}$ of the staining solution for $30 \mathrm{~s}$. The stain was blotted off from the side and grids were air-dried. Grids were imaged using a Thermo Fisher Scientific Talos L120C TEM equipped with a 4K Ceta CEMOS camera using TIA 4.1.5.

\section{Cryo-EM sample preparation}

For the main datasets, purified sample was applied to Quantifoil R2/2, 200 mesh, copper grids floated with an additional approximately $1.1-\mathrm{nm}$ layer of amorphous carbon. Four microlitres of sample was applied onto glow-discharged grids ( $30 \mathrm{~s}$ at $25 \mathrm{~mA}$ ) and allowed to disperse for $60 \mathrm{~s}$ at $4{ }^{\circ} \mathrm{C}$ and $100 \%$ humidity. Grids were blotted for $4-6 \mathrm{~s}$ with a blot force of -5 and plunge-frozen in a liquid propane-ethane mixture, using a Thermo Fisher Scientific Vitrobot Mark V. For the initial Arctica dataset, all steps were similar, with the exception of the additional layer of amorphous carbon.

\section{Cryo-EM data acquisition}

The initial cryo-EM dataset was collected on a 200-kV FEI Talos Arctica electron microscope equipped with a Falcon III direct electron detector running in counting mode and using Thermo Fisher Scientific EPU 1.11. A total of 853 movies were recorded with a nominal magnification of $150,000 \times$, corresponding to a pixel size of 0.96 at the specimen level. Movies were recorded with a total dose of 40.28 electrons per $\mathrm{A}^{2}$, fractionated in 38 frames over a 40 -s exposure time and with a nominal defocus range of $1-2.5 \mu \mathrm{m}$.

The two high-resolution datasets were recorded using Thermo Fisher Scientific EPU 2.4 software on a 300-kV Titan Krios TEM, equipped with a Gatan K3 direct electron detector running in counting mode and a Gatan Bioquantum energy filter (slit size $10 \mathrm{eV}$ ). We recorded 7,984 
and 9,389 movies in counting mode in the two separate sessions with a nominal magnification of $81,000 \times$, corresponding to a pixel size of $1.1 \AA$ at the specimen level. Movies were recorded with a total dose of 59.5 electrons per $A^{2}$, fractionated in 50 frames over a 3 -s exposure time and with a nominal defocus range of $1-3 \mu \mathrm{m}$.

\section{Cryo-EM data processing}

Single-particle analysis was performed using Relion $3.1^{17}$, unless stated otherwise. For the initial Arctica dataset, movies were motion-corrected using MotionCor $2^{18}$ and dose-weighted, and the contrast-transfer function (CTF) was estimated using CTFFIND $4^{19}$. Automated particle-picking was performed using $\mathrm{Cryolo}^{20}$ and the pretrained Janni model. Following particle extraction and several rounds of 2D classification to remove obvious artefacts, an initial de novo model was generated. The dataset was further cleaned using 3D classification and the best class was subsequently used for reference-based particle-picking. Following 2D and 3D classification (and 3D refinement in $\mathrm{C} 1$ ), the map displayed an apparent threefold symmetry and was further refined in C3. This final map displayed an estimated 13.5 Å resolution.

In the first Krios dataset, movies were motion-corrected using MotionCor2, dose-weighted and the CTF was estimated using CTFFIND4. Automated particle-picking was performed using Cryolo with the pretrained Janni model and a low threshold. Particles were extracted and binned $4 \times$ and several rounds of $2 \mathrm{D}$ classification were performed followed by 3D classification with the $30 \AA$ A-filtered Arctica model as a template. The resulting particles were re-extracted without binning, CTF-corrected and polished and refined in $\mathrm{C} 1$, giving a map with an estimated overall resolution of $4.5 \AA$. For the cytosolic region, particles were recentred on the cytosolic region, re-extracted, CTF-corrected, polished and 3D-refined. Following refinement, the density accounting for individual cytosolic dimers was subtracted, resulting in a particle stack that was three times larger. Cytosolic dimers were first refined with a mask encompassing both cytosolic bridges. Subsequently, these were focus-refined using a soft mask around one of the cytosolic bridges. This map was refined using the default Relion value '--tau2fudge 2' but also '--tau2fudge 4', which increased the overall connectivity of the lower cytosolic area. The final map for the cytosolic bridge showed an estimated resolution of $3.3 \AA$ and was sharpened using either Relion postprocessing or DeepEMhancer ${ }^{21}$. DeepEMhancer further helped to improve the observed anisotropy, overall map connectivity.

The second Krios dataset was processed similarly, with some exceptions. Following 3D classification of the binned data against the $4.5 \AA$ Krios map filtered to $30 \AA$, the two maps with and without $\mathrm{MycP}_{5}$ were processed separately. The $\mathrm{MycP}_{5}$-unbound map displayed increased heterogeneity and-following unbinned re-extraction and refinement-the particles were 3D-classified without alignment, resulting in two obvious classes of $4.5 \AA$ and $6.7 \AA$ resolution. Model free density modification in Phenix.Resolve_Cryo_EM ${ }^{22}$ further improved the resolution to $4.3 \AA$ and $5.8 \AA$, respectively. By contrast, a similar 3D classification for the $\mathrm{MycP}_{5}$-bound map did not further classify into structurally distinct classes. Following unbinned re-extraction and refinement, the $\mathrm{MycP}_{5}$-bound map showed an overall resolution of $4 \AA$, which was further improved to $3.8 \AA$ after C3 refinement. Model free density modification in Phenix.Resolve_Cryo_EM further improved the resolution of the entire $\mathrm{C} 1$ map to $3.8 \AA$ and of the $\mathrm{C} 3$ refined map to $3.56 \AA$. For the periplasmic map, the centre of mass for that region was determined using Chimera ${ }^{23}$ and the particles were recentred, extracted, 3D-refined and polished to obtain the periplasmic map at an estimated 3.8 Å resolution in C1. Following 3D classification without alignment and further refinement in $\mathrm{C} 3$, the estimated resolution of the periplasmic map improved to $3.5 \AA$. To separate the two states of $\mathrm{EccC}_{5}$, particles were recentred on the lower cytosolic region, at the level of the DUF domain, polished and 3D-refined. This was followed by a masked 3D classification in which the mask contained NBD1 and $\mathrm{NBD} 2$ of $\mathrm{EccC}_{5}$ in the extended conformation. The two main classes were further 3D-refined unmasked and subsequently masked, leading to a map of about $4.27 \AA$ for the extended conformation and $7.6 \AA$ for the contracted conformation.

\section{Model building and refinement}

Model building started by generating homology models for $\mathrm{MycP}_{5}$, $\mathrm{EccB}_{5}, \mathrm{EccC}_{5}$ and $\mathrm{EccD}_{5}$ with Phyre2 ${ }^{24}$. For $\mathrm{MycP}_{5}$, Protein Data Bank (PDB) entry $4 \mathrm{~J}^{12} 4^{12}$ served as a structural template, and PDB entries $4 \mathrm{KK}^{25}, 4 \mathrm{NHO}^{14}$ and $6 \mathrm{SGW}^{6}$, and $6 \mathrm{SGZ}^{6}$ served as reference models for $\mathrm{EccB}_{5}, \mathrm{EccC}_{5}$ and $\mathrm{EccD}_{5}$, respectively. To obtain atomic models of the periplasmic part $\left(\mathrm{MycP}_{5}-\mathrm{EccB}_{5}\right)$ of the M. tuberculosis ESX-5 complex, homology models of $\mathrm{MycP}_{5}$ and $\mathrm{EccB}_{5}$ were rigid-body-fitted into a $\mathrm{C} 1$ symmetry, focus-refined periplasmic $M$. tuberculosis ESX-5 map (Electron Microscopy Data Bank (EMDB) code EMD-12518) using the fit-in-map tool in ChimeraX (v.1.0 ${ }^{26}$. Model building, extension and interactive refinement was performed with ISOLDE (v.1.0.1) ${ }^{27}$, a molecular-dynamics-guided structure refinement tool within ChimeraX (v.1.0). The resulting coordinate file (PDB 7NPS) was further refined with Phenix.real_space_refine (v.1.18.2-3874) ${ }^{28}$ using reference model restraints, strict rotamer matching and disabled grid search. Model validation was carried out using the MolProbity web server ${ }^{29}$ and EMRinger ${ }^{30}$ within the Phenix software package. Models for the membrane-embedded region $\left(\mathrm{MycP}_{5}-\mathrm{EccB}_{5}-\mathrm{EccC}_{5}-\mathrm{EccD}_{5}\right)$ and cytoplasmic bridge (cytosolic domains of $\mathrm{EccC}_{5}-\mathrm{EccD}_{5}$ ) (PDB 7NPT) were built in the same way, using a reconstruction of the full $M$. tuberculosis ESX-5 complex (EMDB EMD-12517) and a focus-refined map of the cytoplasmic domains (EMDB EMD-12520) sharpened with DeepEMhancer, respectively. Finally, a composite model was assembled by fusing the periplasmic assembly and six copies of the cytosolic bridge to the membrane-embedded region model. This composite model was then refined against the full $M$. tuberculosis ESX-5 complex map with C1 symmetry (PDB 7NP7 and EMDB EMD-12514) and C3 symmetry (PDB 7NPR and EMDB EMD-12517).

Modelling into $\mathrm{MycP}_{5}$-free maps was performed with ISOLDE using the composite ESX-5 model, in which $\mathrm{MycP}_{5}$ and the periplasmic domain of $\mathrm{EccB}_{5}$ (residues 84-507) had been deleted. Adaptive distance restraints as well as torsion restraints were applied to all atoms to restrain short-range conformational changes but allow for long-range conformational movements. ISOLDE simulations for dynamic fitting of the coordinate file into EMD-12521 and EMD-12522 were performed (about $10 \mathrm{~min}, 10 \mathrm{~K}$ ) after which the models showed satisfying fits to the new maps without further manual intervention. $\mathrm{MycP}_{5}$-free models were further refined against the maps using Phenix.real_space_refine (v.1.18.2-3874) as stated.

Visualization of atomic coordinates and map volumes was performed with ChimeraX (v.1.1) and PyMOL v.2.40 ${ }^{31}$. Buried surface areas between subunits were calculated by PISA $^{32}$.

\section{Reporting Summary}

Further information on research design is available in the Nature Research Reporting Summary linked to this article.

\section{Data availability}

Cryo-EM maps have been deposited in the EMDB under accession codes EMD-12514 (full complex in C1), EMD-12517 (full complex in C3), EMD12518 (periplasmic map in C1), EMD-12519 (periplasmic map in C3), EMD-12520 (cytosolic bridge), EMD-12521 ( MycP $_{5}$-free map 1), EMD$12522\left(\mathrm{MycP}_{5}\right.$-free map 2), EMD-12523 ( $\mathrm{EccC}_{5}$, extended state) and EMD12525 ( $\mathrm{EccC}_{5}$, contracted state). The composite model settled in the $\mathrm{C} 1$ and $\mathrm{C} 3$ full maps, periplasm in $\mathrm{C} 1$, cytosolic bridge, $\mathrm{MycP}_{5}$-free map 1 and $\mathrm{MycP}_{5}$-free map 2 have been deposited in the PDB under accession codes 7NP7, 7NPR, 7NPS, 7NPT, 7NPU and 7NPV, respectively. All other data are available from the corresponding author upon reasonable request. 
16. Houben, E. N. et al. Composition of the type VII secretion system membrane complex Mol. Microbiol. 86, 472-484 (2012).

17. Zivanov, J., Nakane, T. \& Scheres, S. H. W. Estimation of high-order aberrations and anisotropic magnification from cryo-EM data sets in RELION-3.1. IUCrJ 7, 253-267 (2020).

18. Zheng, S. Q. et al. MotionCor2: anisotropic correction of beam-induced motion for improved cryo-electron microscopy. Nat. Methods 14, 331-332 (2017).

19. Rohou, A. \& Grigorieff, N. CTFFIND4: fast and accurate defocus estimation from electron micrographs. J. Struct. Biol. 192, 216-221 (2015).

20. Wagner, T. \& Raunser, S. The evolution of SPHIRE-crYOLO particle picking and its application in automated cryo-EM processing workflows. Commun. Biol. 3, 61 (2020).

21. Sanchez-Garcia, R. et al. DeepEMhancer: a deep learning solution for cryo-EM volume post-processing. Preprint at https://doi.org/10.1101/2020.06.12.148296 (2020).

22. Terwilliger, T. C., Ludtke, S. J., Read, R. J., Adams, P. D. \& Afonine, P. V. Improvement of cryo-EM maps by density modification. Nat. Methods 17, 923-927 (2020).

23. Pettersen, E. F. et al. UCSF Chimera-a visualization system for exploratory research and analysis. J. Comput. Chem. 25, 1605-1612 (2004)

24. Kelley, L. A., Mezulis, S., Yates, C. M., Wass, M. N. \& Sternberg, M. J. The Phyre2 web portal for protein modeling, prediction and analysis. Nat. Protocols 10, 845-858 (2015).

25. Wagner, J. M. et al. Structures of EccB1 and EccD1 from the core complex of the mycobacterial ESX-1 type VII secretion system. BMC Struct. Biol. 16, 5 (2016).

26. Pettersen, E. F. et al. UCSF ChimeraX: structure visualization for researchers, educators, and developers. Protein Sci. 30, 70-82 (2021).

27. Croll, T. I. ISOLDE: a physically realistic environment for model building into low-resolution electron-density maps. Acta Crystallogr. D 74, 519-530 (2018).

28. Liebschner, D. et al. Macromolecular structure determination using X-rays, neutrons and electrons: recent developments in Phenix. Acta Crystallogr. D 75, 861-877 (2019).

29. Williams, C. J. et al. MolProbity: more and better reference data for improved all-atom structure validation. Protein Sci. 27, 293-315 (2018).

30. Barad, B. A. et al. EMRinger: side chain-directed model and map validation for 3D cryo-electron microscopy. Nat. Methods 12, 943-946 (2015).

31. The PyMOL Molecular Graphics System v.1.8 (Schrödinger, 2015).

32. Krissinel, E. \& Henrick, K. Inference of macromolecular assemblies from crystalline state. J. Mol. Biol. 372, 774-797 (2007).
Acknowledgements We thank all members of the laboratories of T.C.M., E.N.G.H. and W.B. for their support of this project; W. Lugmayr for scientific IT support; L. Ciccarelli for initial cryo-EM analysis of the M. tuberculosis ESX-5 complex; and T. Croll for his support with ISOLDE. High-performance computing was possible through access to the HPC at DESY/Hamburg. Cryo-EM data collection was performed at the Cryo-EM Facility at CSSB. This project was supported by funds available to T.C.M. through the Behörde für Wissenschaft, Forschung und Gleichstellung of the city of Hamburg at the Institute of Structural and Systems Biology at the University Medical Center Hamburg-Eppendorf (UKE). The laboratory of T.C.M. is supported by DESY (German Electron Synchrotron Center). The cryo-EM facility is supported by the University of Hamburg, the University Medical Center Hamburg-Eppendorf and DFG grant numbers INST152/772-1, 152/774-1, 152/775-1, 152/776-1 and 152/777-1 FUGG. This work received funding by a VIDI grant (864.12.006; to C.M.B. and E.N.G.H.) from the Netherlands Organization of Scientific Research. This project has received funding from the European Union's Horizon 2020 research and innovation programme under the Marie Sklodowska-Curie grant agreement no. 101030373 (to C.M.B.)

Author contributions C.M.B., D.F., J.W., E.N.G.H., W.B. and T.C.M. designed experiments. R.U. and C.M.B. generated constructs. C.M.B. purified complexes and performed biochemical assays. J.W. vitrified samples and collected cryo-EM images. C.M.B. and J.W. collected negative-stain images. D.F. built the atomic model. C.M.B., D.F., E.N.G.H., W.B. and T.C.M. interpreted data. C.M.B. and T.C.M. processed cryo-EM data. C.M.B., D.F., E.N.G.H., W.B. and T.C.M. wrote and revised the paper. All authors read, corrected and approved the manuscript. E.N.G.H., W.B. and T.C.M. supervised the project.

Funding Open access funding provided by Deutsches Elektronen-Synchrotron (DESY).

Competing interests The authors declare no competing interests.

\section{Additional information}

Supplementary information The online version contains supplementary material available at https://doi.org/10.1038/s41586-021-03517-z.

Correspondence and requests for materials should be addressed to T.C.M.

Peer review information Nature thanks the anonymous reviewers for their contribution to the peer review of this work. Peer reviewer reports are available.

Reprints and permissions information is available at http://www.nature.com/reprints. 
a

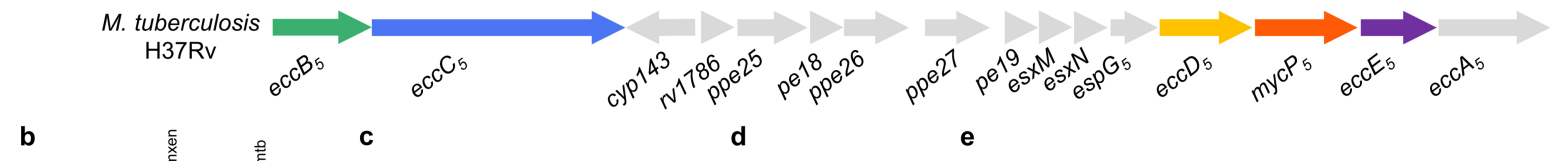

b

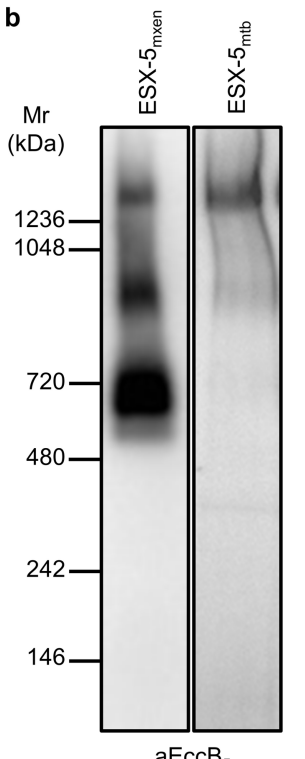

f

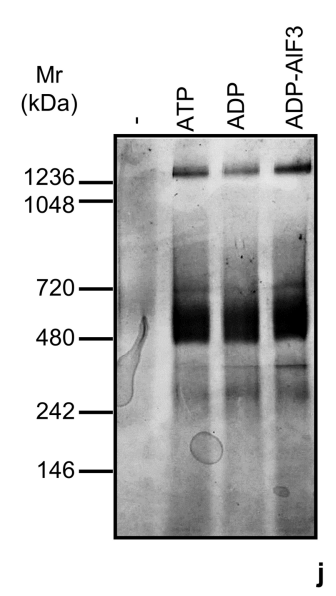

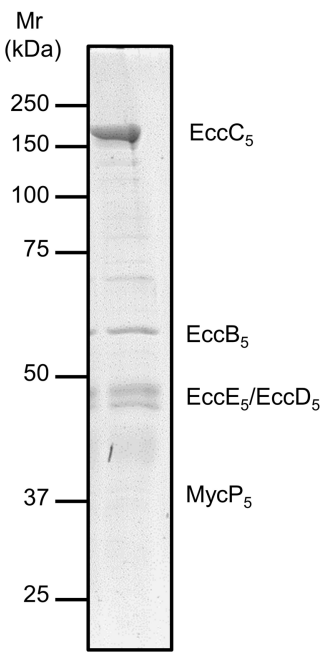

g

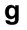

$\mathrm{Mr}$

(kDa)
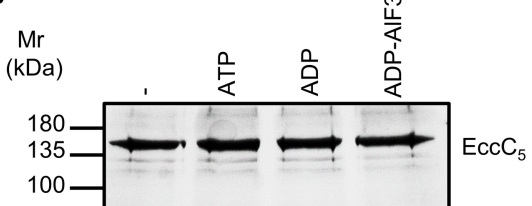

75
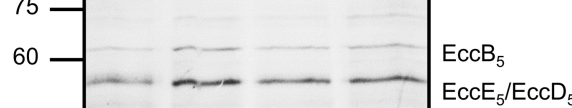

$37-1$

25

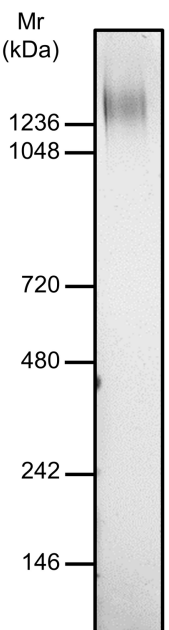

h

$\mathrm{Mr}$

$(\mathrm{kDa})$

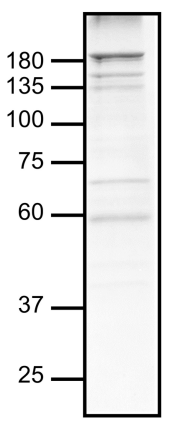

i

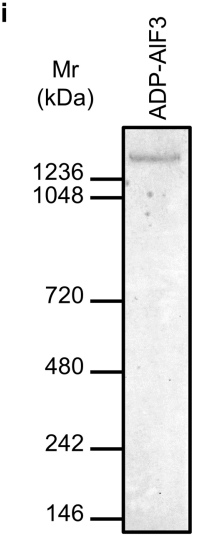

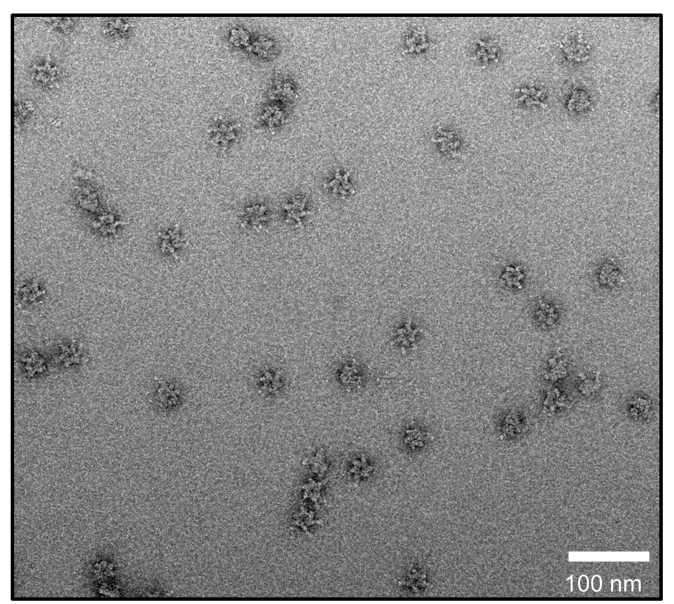

Extended Data Fig. 1 | See next page for caption. 
Extended Data Fig. 1 | Purification of the M. tuberculosis ESX-5 membrane complex. a, Genetic organization of the esx-5locus of M.tuberculosis H37Rv, which has been cloned and expressed in M. smegmatis MC $^{2} 155$. b, BN-PAGE and western blot analysis using an anti- $\mathrm{EccB}_{5}$ antibody of DDM-solubilized membranes from M. smegmatis $\mathrm{MC}^{2} 155$ expressing M.xenopi or M. tuberculosis ESX-5 (ESX-5 mxen $_{\text {or }}$ ESX-5 ${ }_{\mathrm{mtb}}$, respectively). Experiment was reproduced three times. c, d, Coomassie-stained SDS-PAGE (c) and BN-PAGE (d) of Strep- and SEC-purified ESX-5 $5_{\text {mtb }}$ membrane complexes. e, Negative-stain electron microscopy analysis of ESX-5 $5_{\text {mtb }}$ membrane complexes shown in $\mathbf{c}$ and $\mathbf{d}$. Experiments in $\mathbf{c}-\mathbf{e}$ were replicated three times.f, BN-PAGE and Coomassie staining of Strep-purified ESX $-5_{\text {mtb }}$ complexes without nucleotides $(-)$ or in the presence of nucleotides ATP, ADP or the transition-state analogue ADP-AIF . $_{\text {. }}$ Upon purification, in the presence of either nucleotide, the higher-molecularweight species of the membrane complex becomes more prominent. $\mathbf{g}$, SDS-PAGE and Coomassie staining of the same samples as in $\mathbf{f}$, showing a similar SDS-PAGE protein pattern between the four conditions. Experiment in $\mathbf{f}, \mathbf{g}$ was performed three times. $\mathbf{h}, \mathbf{i}$, Coomassie-stained SDS-PAGE (h) and BN-PAGE (i) of Strep- and SEC-purified ESX-5 $5_{\text {mtb }}$ membrane complexes in the presence of $\mathrm{ADP}-\mathrm{AlF}_{3} . \mathbf{j}$, Negative-stain electron microscopy of the same sample as in $\mathbf{h}, \mathbf{i}$, showing improved sample homogeneity as compared to purifications in the absence of nucleotides, as shown in e. Experiment shown in $\mathbf{h}-\mathbf{j}$ was performed twice. 


\section{Article}

a

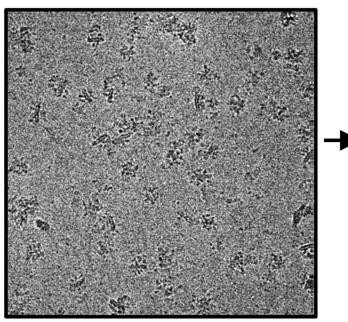

853 micrographs

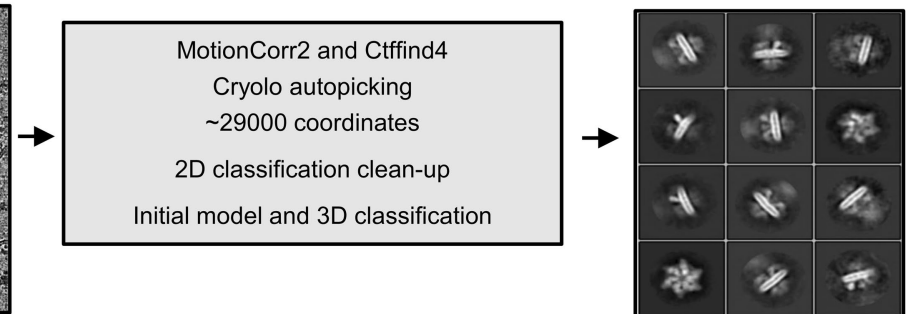

Cryolo autopicking

29000 coordinates

2D classification clean-up

Initial model and 3D classification
Reference based particle picking 2D classification 3D classification $3 \mathrm{D}$ refinement in $\mathrm{C} 1$ and $\mathrm{C} 3$

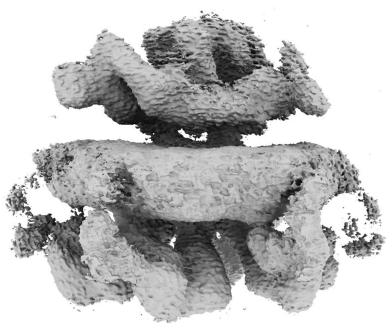

C1

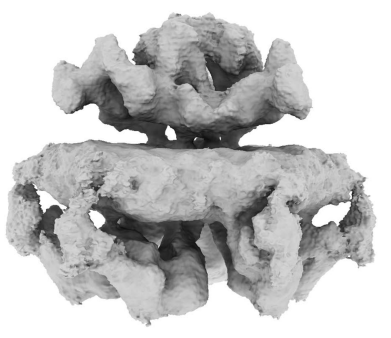

C3 - $13.5 \AA$

b
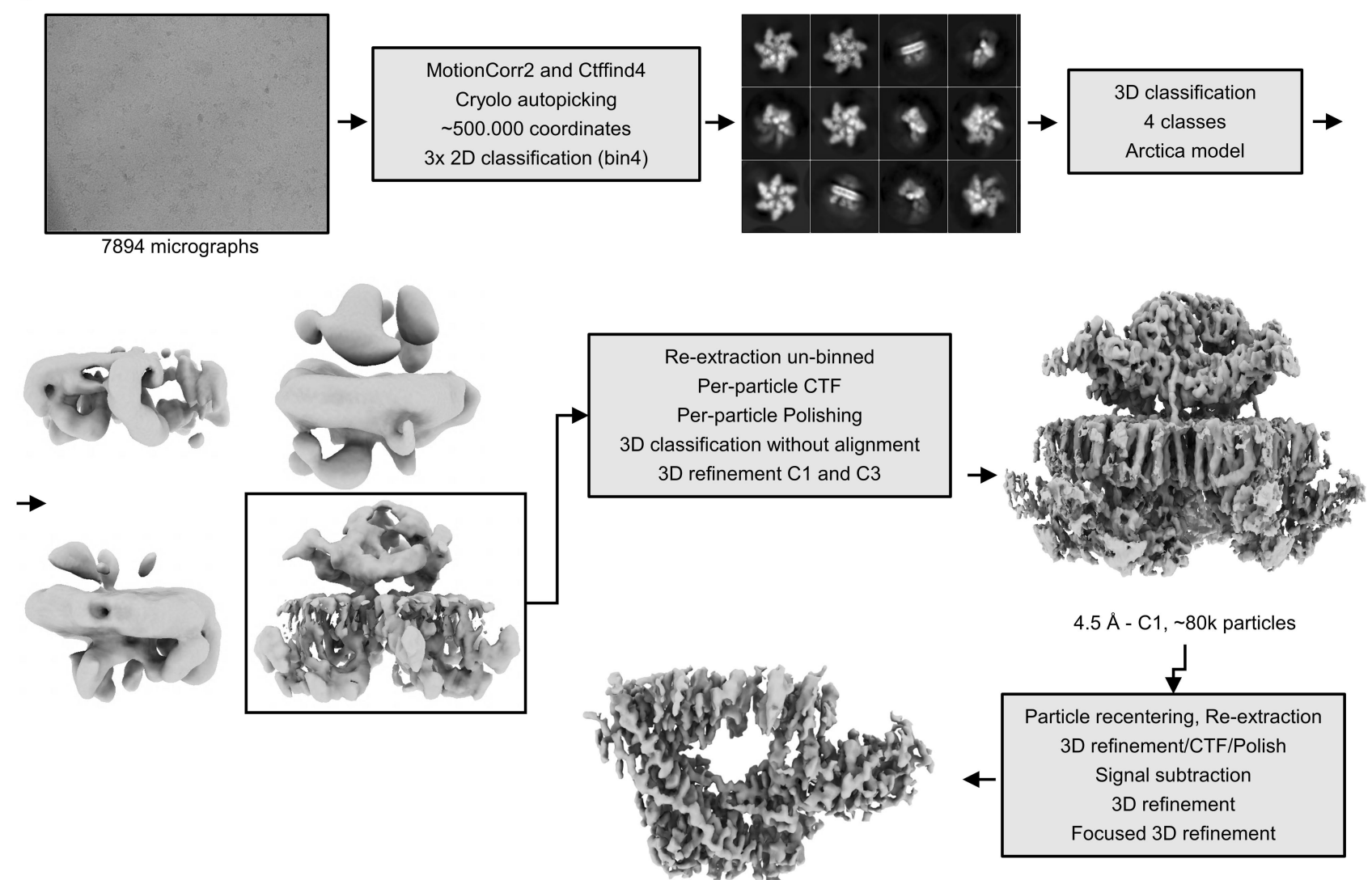

$\sim 4500$ particles

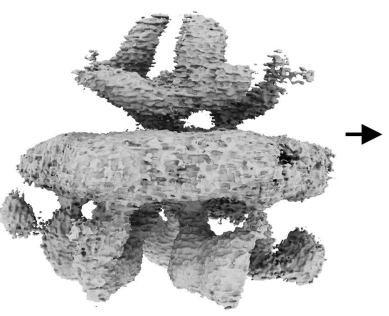

1100 particles

$3.3 \AA, \sim 240.000$ particles

Extended Data Fig. 2 |Cryo-EM data collection and single-particle reconstruction procedure. a, b, This figure relates to the initial Talos-Arctica-collected dataset (a) and the first higher-resolution Titan-Krios-collected dataset (b). 


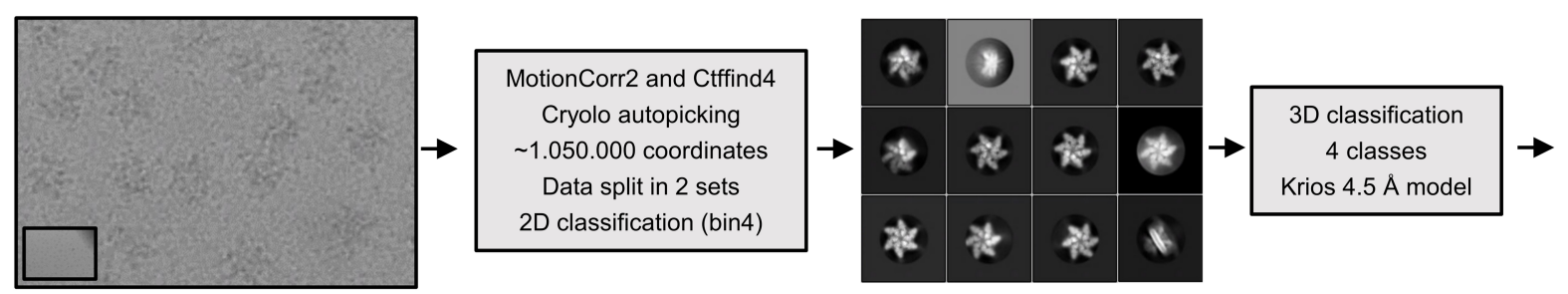

8256 micrographs
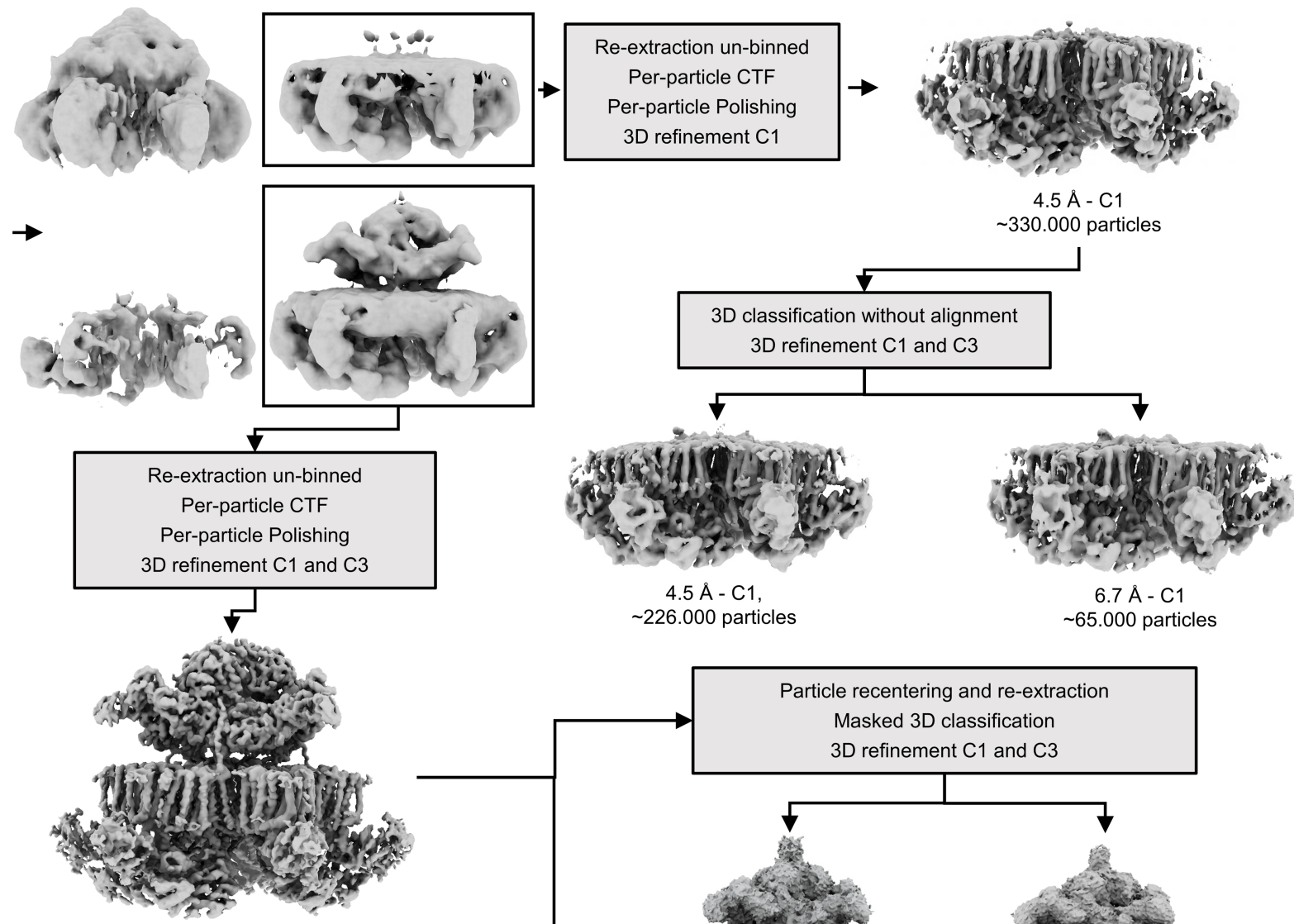

$\sim 226.000$ particles

$\sim 65.000$ particles

$4 \AA, \sim 155.000$ particles

Particle recentering on periplasm center of mas Re-extraction $3 \mathrm{D}$ refinement/CTF/Polish Signal subtraction 3D refinement $\mathrm{C} 1$ and $\mathrm{C} 3$
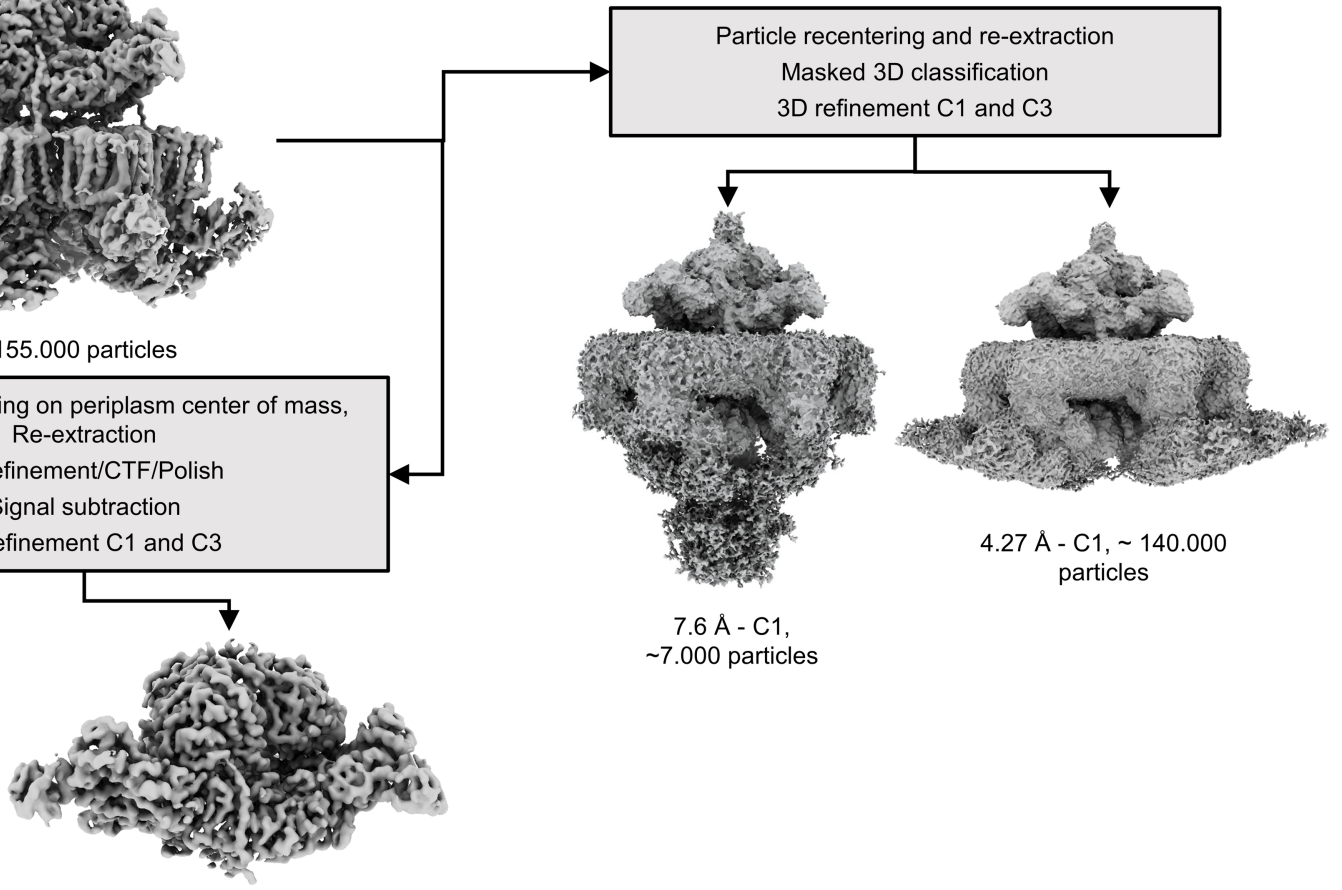

$7.6 \AA-\mathrm{C} 1$

$\sim 7.000$ particles

$3.8 \AA-C 1$

$\sim 155.000$ particles

Extended Data Fig. 3 |Cryo-EM data collection and single-particle reconstruction procedure. This figure relates to the second high-resolution Titan Krios collected dataset. 

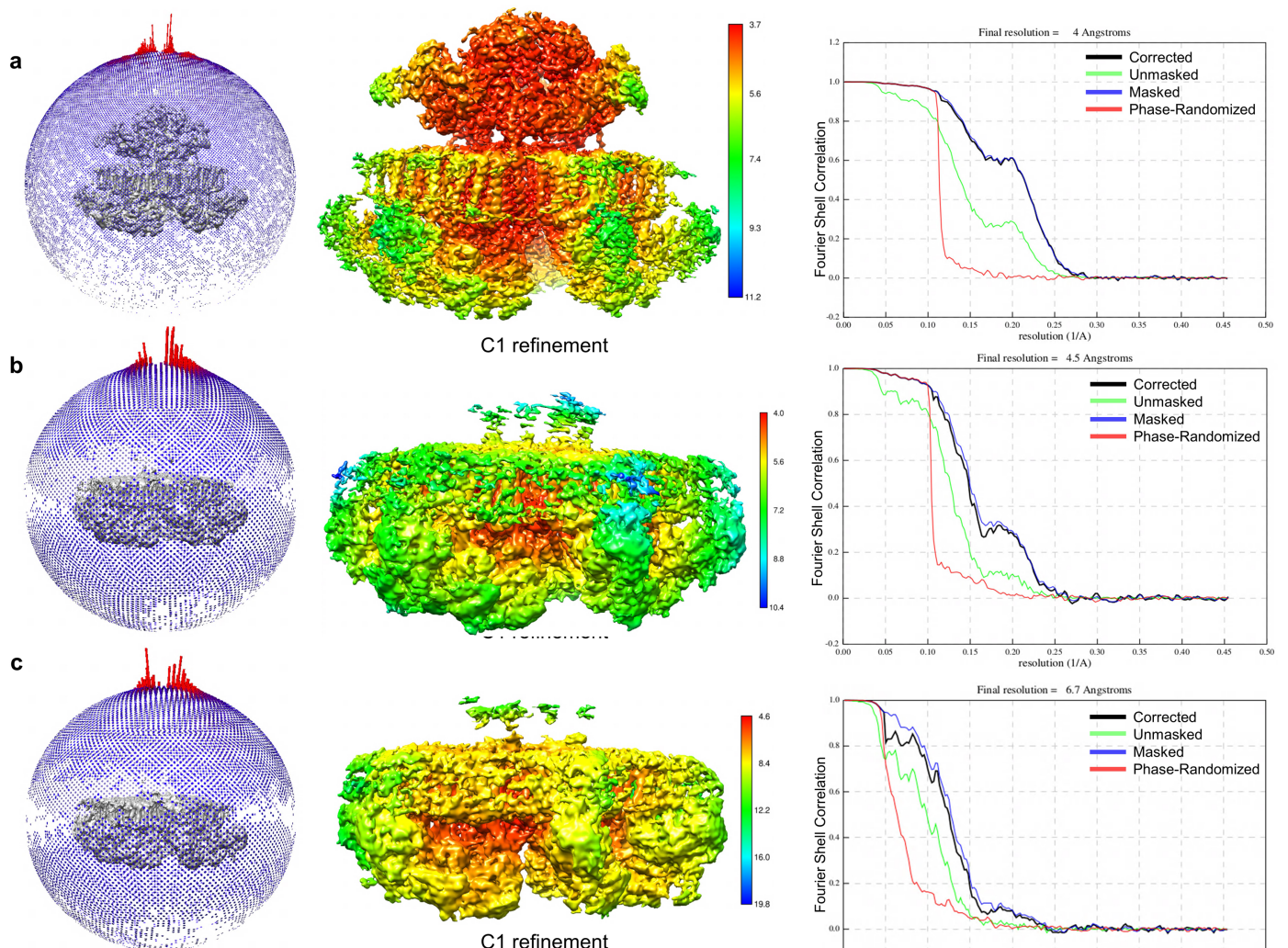

C1 refinement

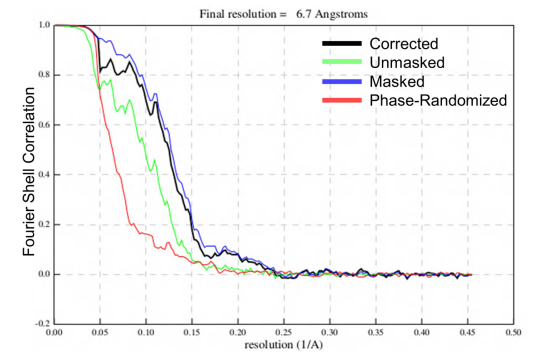

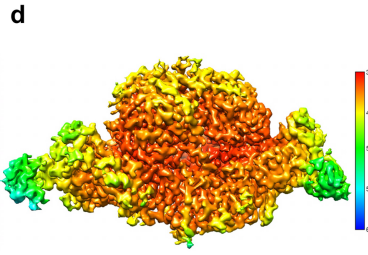

C1 refinement

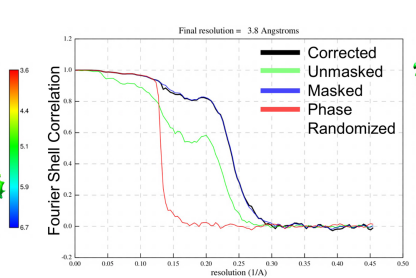

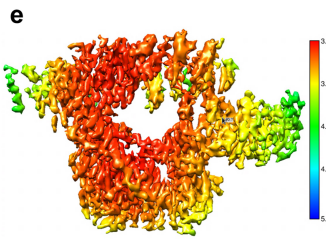

C1 refinement
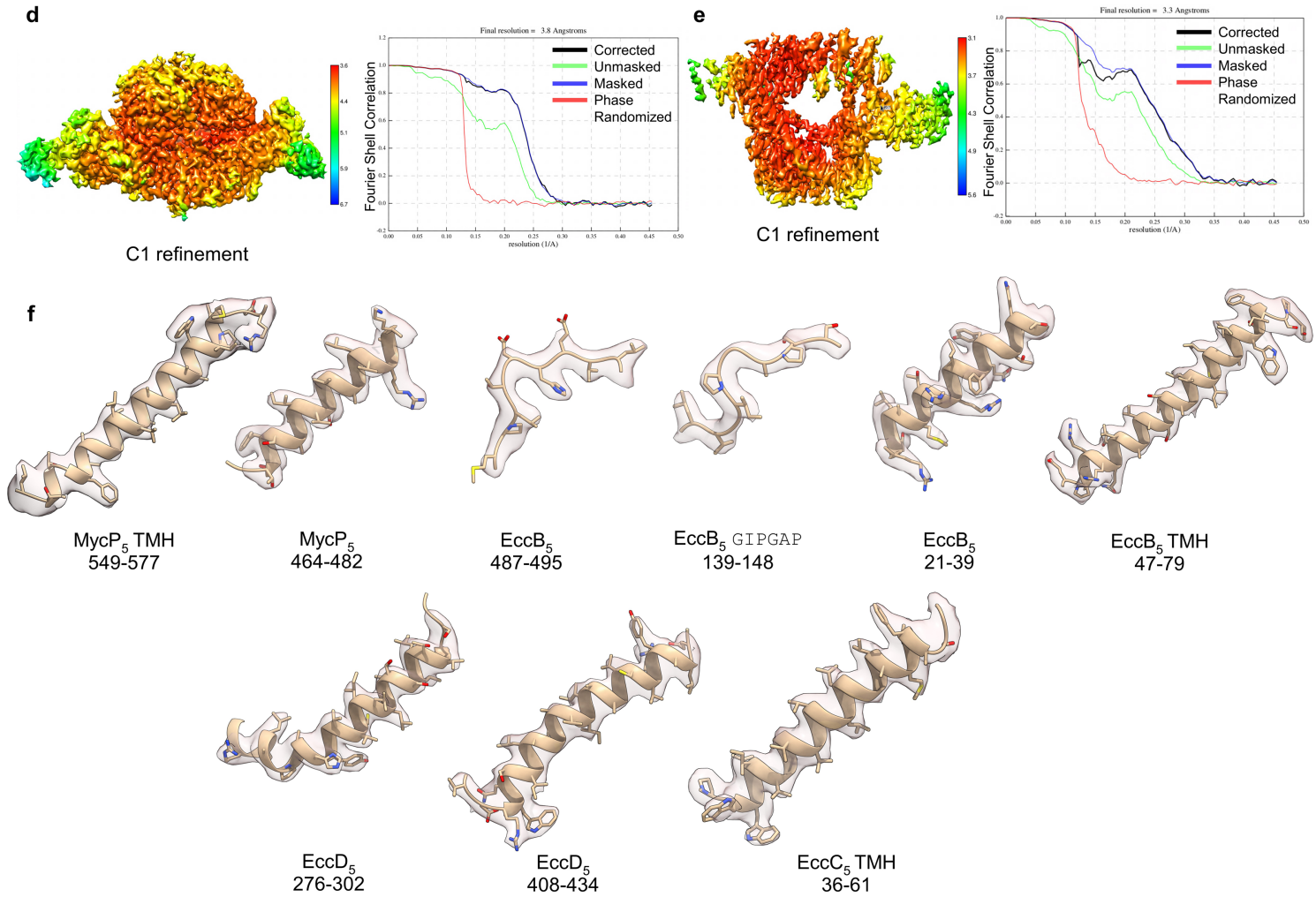

$\mathrm{CCB}_{5}$ GIPGAP

$\mathrm{EccB}_{5}$

$\mathrm{EccB}_{5} \mathrm{TMH}$

$139-148$

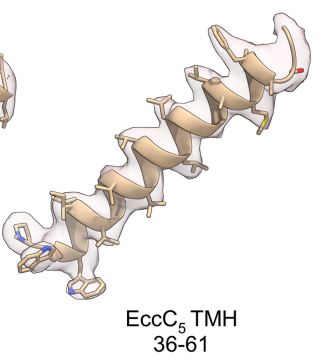

Extended Data Fig. 4 | Single-particle reconstructions of the ESX-5 $5_{\text {mtb }}$ membrane complex. a-c, Angular distribution plots, local-resolution estimations and Fourier shell correlation (FSC) plots of the $\mathrm{C} 1$ reconstruction of the entire $\mathrm{MycP}_{5}$-bound ESX-5 ${ }_{\mathrm{mtb}}$ membrane complex (a), and C1 reconstructions of the two heterogeneous $\mathrm{MycP}_{5}$-unbound ESX- $5_{\text {mtb }}$ membrane complexes (b, c).d, e, Local-resolution estimation and FSC plot for the C1-refined periplasmic map (d) and the map of the cytosolic bridge (e). f, Examples of cryo-EM densities and corresponding models. 


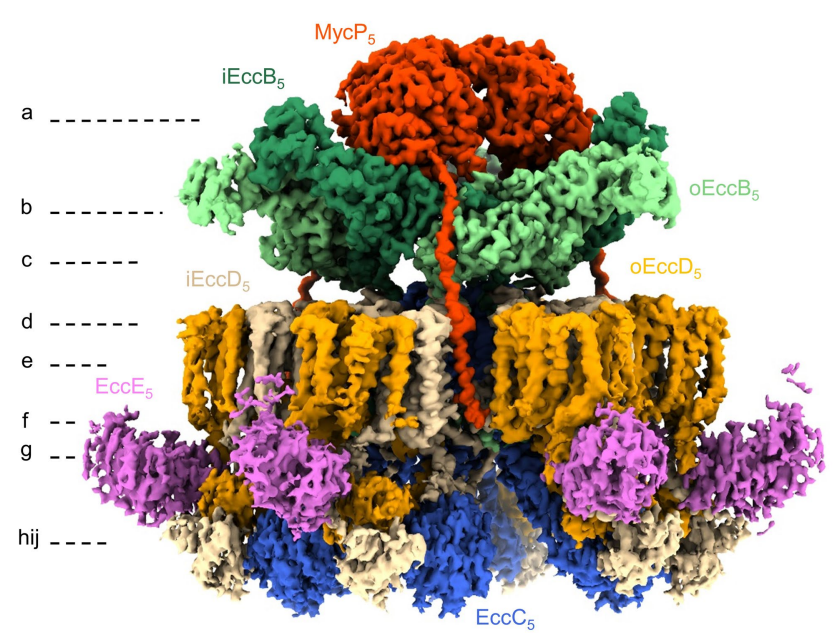

c
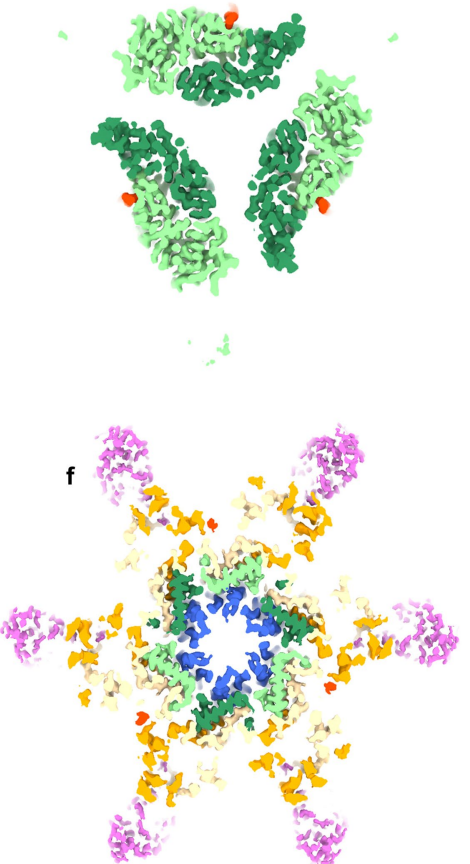

d
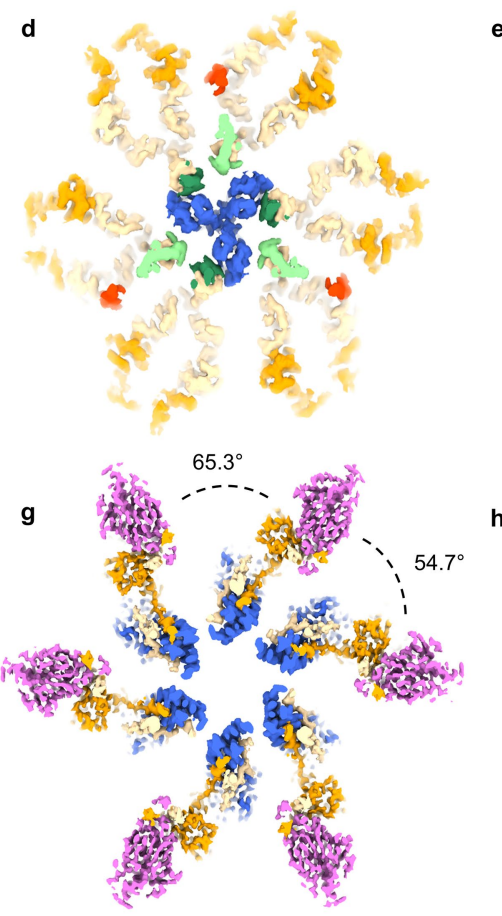

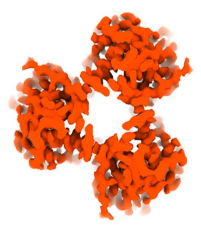

b

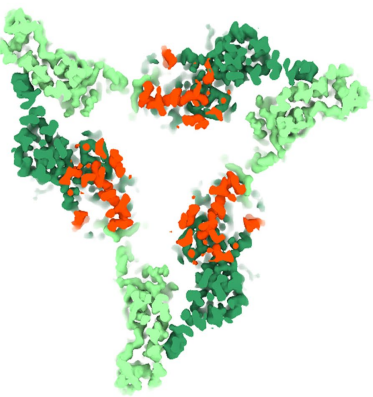

e tकी

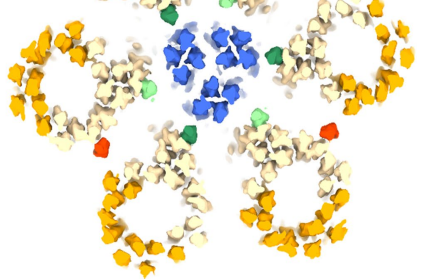

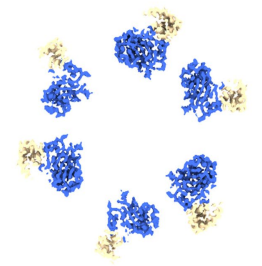

Extended Data Fig. 5 | See next page for caption.

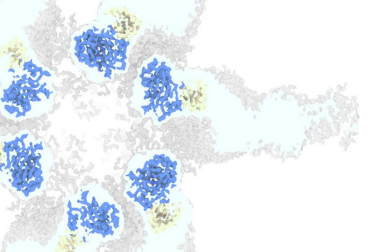




\section{Article}

Extended Data Fig. 5 | Top cross-sections through the intact ESX-5 $5_{\text {mtb }}$

membrane complex. a, $\mathrm{MycP}_{5}$ trimer top view, highlighting the pore formed at the periplasmic side. b, Section through the periplasmic assembly at the $\mathrm{EccB}_{5}{ }^{-}$ $\mathrm{Myc}_{5}$ interface, showing the position of the protease domain sitting on top of inner $\mathrm{EccB}_{5} . \mathbf{c}$, Section through the periplasmic assembly at the $\mathrm{EccB}_{5}$ dimer interface level, highlighting the $\mathrm{MycP}_{5}$ linker connection to the TMH. d, Top view of the six membrane protomers with the closed $\mathrm{EccC}_{5} \mathrm{TMH}$ pyramid at the centre. e, Top cross-section through the six membrane protomers, highlighting 153 of the 165 TMHs. At the central area towards the cytosol, the three-EccC ${ }_{5} \mathrm{TMH}$ pyramid opens up in a manner similar to an iris. $\mathrm{MycP}_{5}$, the protease domain of which interacts with the protomer containing inner $\mathrm{EccB}_{5}$, interacts with the outer $\mathrm{EccD}_{5}$ barrel of the adjacent protomer at the membrane level. At the membrane level, the angle between protomers within a dimer and between adjacent protomers of different dimers differs by only $0.5^{\circ}$.f, Top section displaying the region below the inner leaflet of the inner membrane, highlighting a further opening of the $\mathrm{EccC}_{5}$ gated pore and the lower part of the $\mathrm{EccB}_{5}$ basket, formed by $\mathrm{EccB}_{5} \mathrm{~N}$ termini. $\mathrm{g}$, At the cytosolic level, the angle between protomers differs to that at the membrane level. As such, the angle between protomers within a dimer grows to $65.3^{\circ}$, while the angle between adjacent protomers of different dimers decreases to $54.7^{\circ}$. The change in angles between the membrane and cytosolic regions of protomers is caused by $\mathrm{MycP}_{5}$ binding, which induces a slight tilting to the protomers that it binds via inner $\mathrm{EccD}_{5} \cdot \mathbf{h}$, Section through the lower region of the cytosolic bridge, containing the DUF domain of $\mathrm{EccC}_{5}$ and the cytosolic domain of inner $\mathrm{EccD}_{5} . \mathbf{i}$, Same view as in $\mathbf{h}$, but overlaid with the $\mathrm{EccC}_{5}$ extended state, highlighting the radial extension of the EccC ${ }_{5}$ NBD1, NBD2 and NBD3 almost parallel to the inner membrane. $\mathbf{j}$, Same view as in $\mathbf{h}$, but then overlaid with the $\mathrm{EccC}_{5}$ contracted stated. 
a
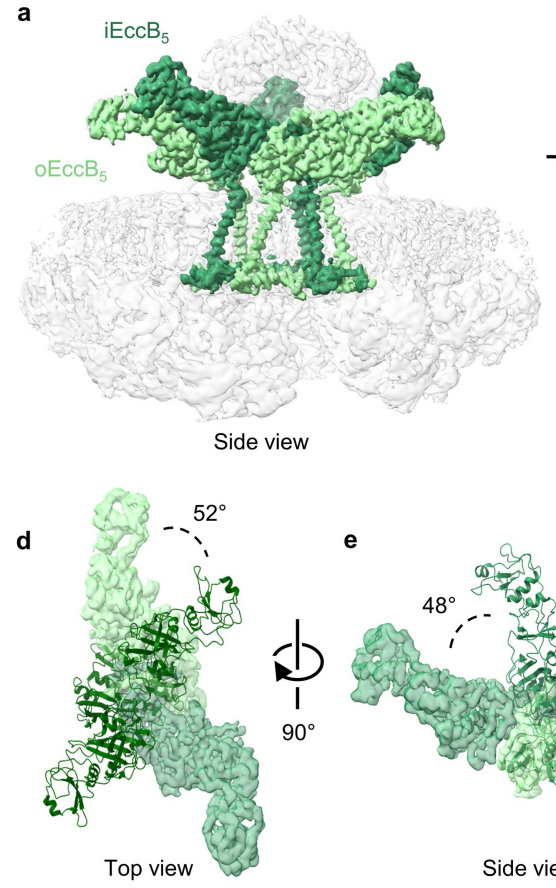

g

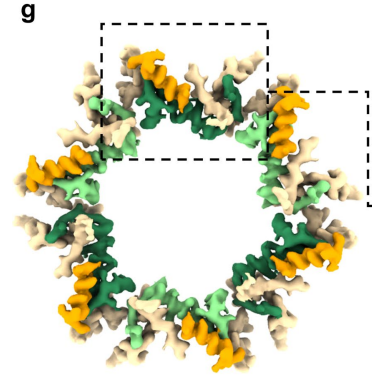

Bottom view

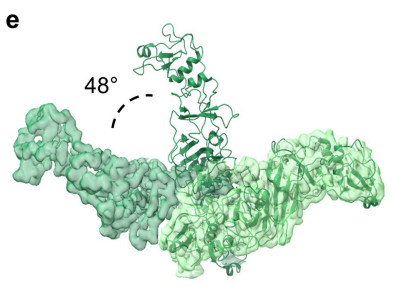

Side view

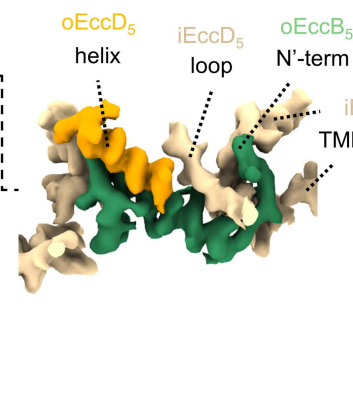

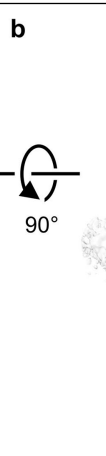

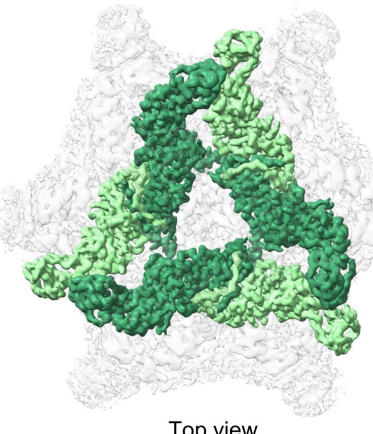

Top view
C

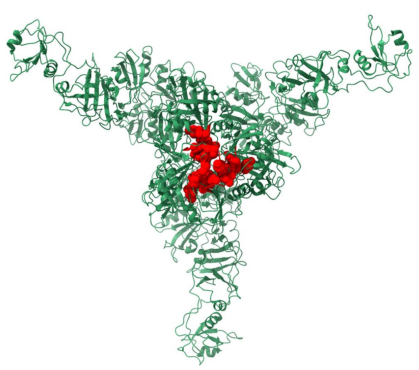

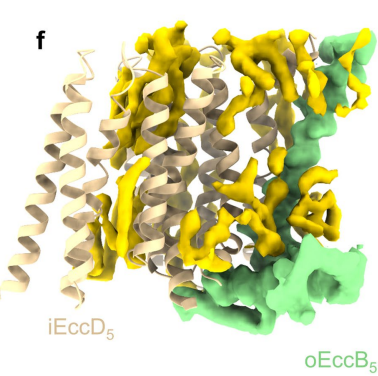

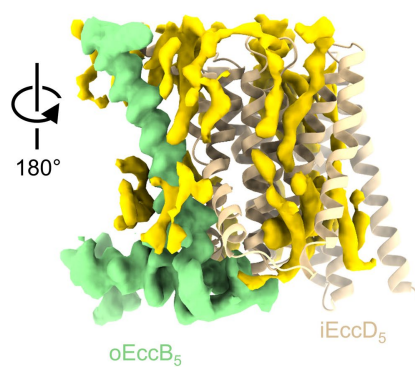

h

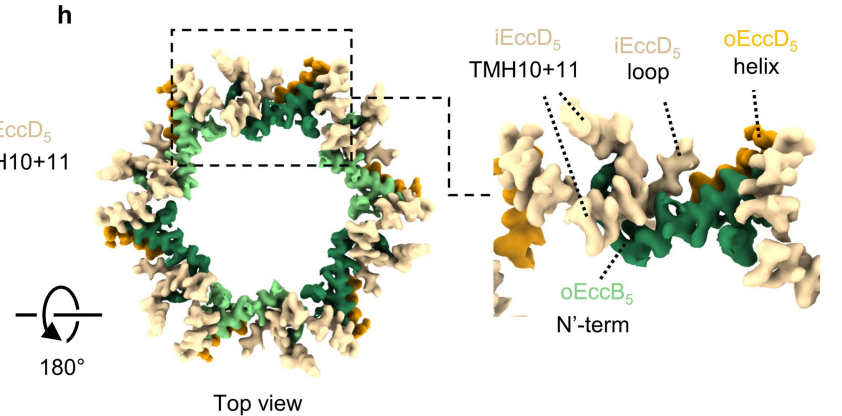

i

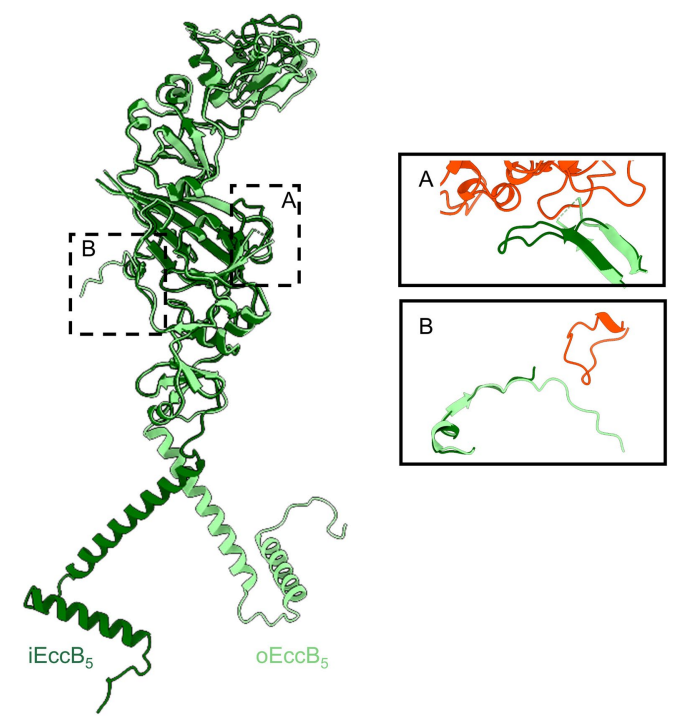

Extended Data Fig. 6 |See next page for caption. 


\section{Article}

Extended Data Fig. 6 | Hexameric EccB $_{5}$ adopts a triangular conformation in the periplasm. a, b, Side (a) and top (b) view of an intact ESX- $5_{\mathrm{mtb}}$ assembly in which inner $\mathrm{EccB}_{5}$ and outer $\mathrm{EccB}_{5}$ are coloured as in Fig. 1 and the rest of the components are transparent. c, A V-shaped $\mathrm{EccB}_{3}$ dimer (PDB 6SGY) was fitted into the $M$. smegmatis ESX-3 dimer cryo-EM density (EMDB EMD-20820) together with the corresponding dimeric ESX-3 model for the membrane and cytosolic domains (PDB 6UMM) using the Chimera fit in map tool. This composite dimer model was subsequently trimerized, on the basis of our full ESX-5 map reconstructions. The clashing of $\mathrm{EccB}_{3}$ periplasmic domains between the dimers, towards the central area, in this hybrid model are highlighted in red. d, Upon $\mathrm{Myc}_{5}$ binding to the assembly, the periplasmic $\mathrm{EccB}_{5}$ dimer is rotated by $52^{\circ}$, avoiding the clashes observed in c. Angles were measured by aligning the hybrid model and the ESX-5 $5_{\mathrm{mtb}}$ model at the membrane level. Subsequently, centres of mass were defined for the combined R1 domains of each $\mathrm{EccB}_{3}$ and $\mathrm{EccB}_{5}$ dimer (at the base of the dimer) and for every R2 and R3 EccB monomer (toward the tips of the EccB dimer). Planes defined by these three points were generated for both $\mathrm{EccB}_{3}$ and $\mathrm{EccB}_{5}$ dimers and angles were measured between these two planes. $\mathrm{EcCB}_{3}$ dimer is shown as a ribbon model and $\mathrm{EccB}_{5}$ model is shown as zoned density. e, Compared to the
V-shaped $\mathrm{EccB}_{3}$ dimer (ribbon model), the angle between the two $\mathrm{EccB}_{5}$ monomers (zoned density) grows by $48^{\circ}$ upon $\mathrm{MycP}_{5}$ binding. EccB dimer angles were calculated by measuring the angle between the centres of mass of the R2 and R3 domains of each EccB protomer in relation to the centre of mass of both R1 domains. $f$, Side views of the $\mathrm{TMH}$ region of inner $\mathrm{EccD}_{5}$, depicted as a ribbon model, and the TMH and $\mathrm{N}$ terminus of an interacting outer $\mathrm{EccB}_{5}$, depicted as zoned density. An array of lipids found in the $\mathrm{EccD}_{5}$ barrel (but also surrounding this inner $\mathrm{EcCD}_{5}-\mathrm{EccB}_{5}$ interaction site) are depicted in gold. g, Bottom view of the lower cytosolic area of the $\mathrm{EccB}_{5}$ basket, formed by EccB $\mathrm{N}$ termini (residues 10-48) and depicted with the interacting pocket formed by TMH10, TMH11 and TMH8 (not shown for clarity) of inner $\mathrm{EcCD}_{5}$ of the adjacent protomer. The $\mathrm{EccB}_{5} \mathrm{~N}$ terminus is also buttressed in this position by a short helix (residues 119-130) of outer $\mathrm{EccD}_{5}$, which connects outer $\mathrm{EccD}_{5} \mathrm{TMHs}_{\mathrm{H}}$ with its cytosolic domain, and also by part of the inner EcCD $_{5}$ loop (residues 307-315) that subsequently folds along the stalk and DUF domain of $\mathrm{EccC}_{5} \cdot \mathbf{h}$, Same map as in $\mathbf{g}$, but viewed from the top. $\mathbf{k}$, Superposition of inner $\mathrm{EccB}_{5}$ and outer $\mathrm{EccB}_{5}$, highlighting conformational differences between the two, which are the result of the interaction with $\mathrm{MycP}_{5}$. 


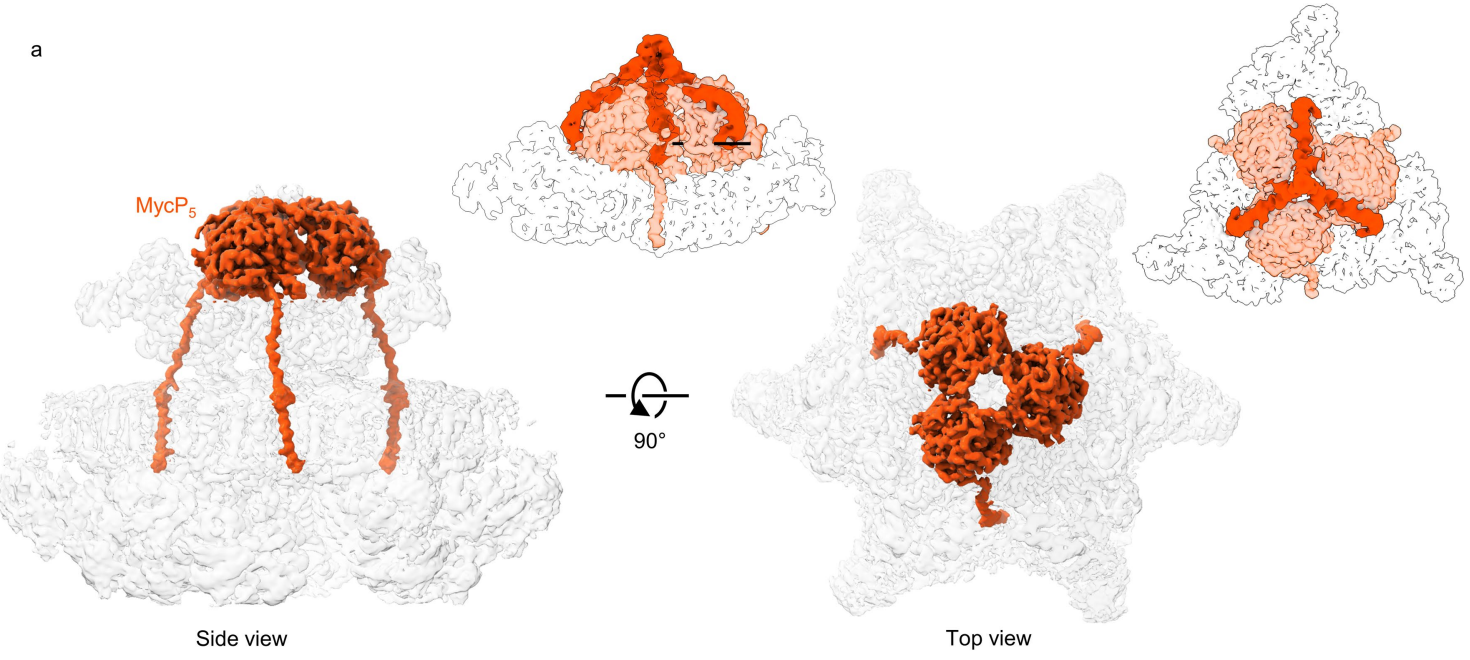

b

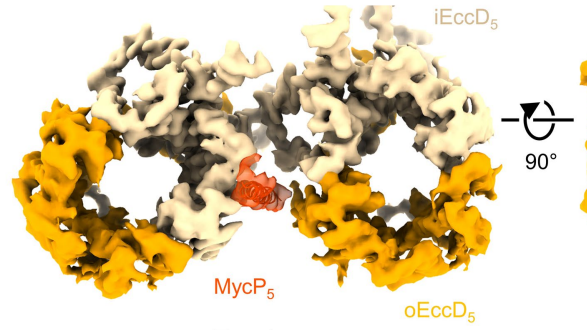

Top view

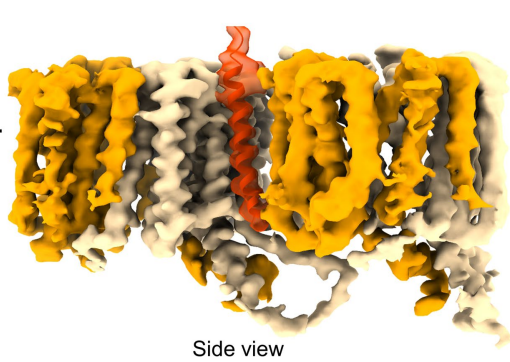

c
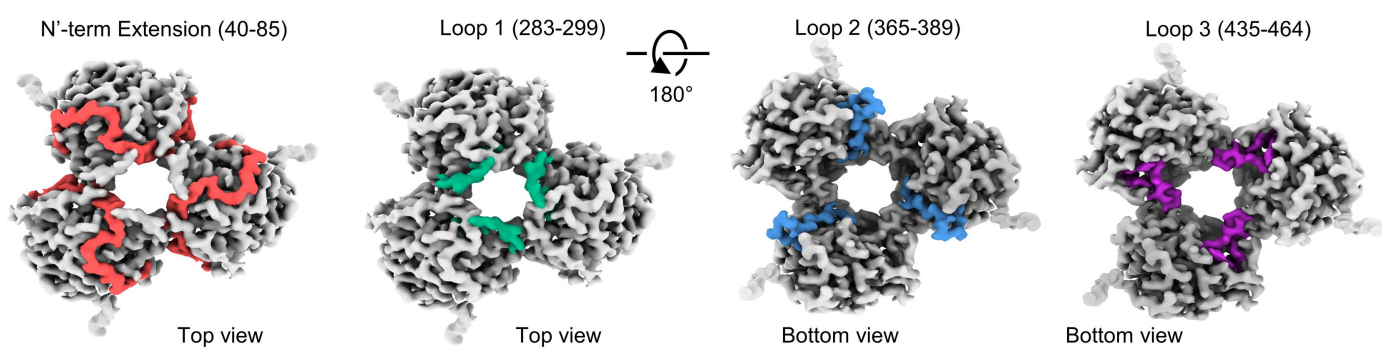

Loop 4 (337-343)

Loop 5 (151-276)
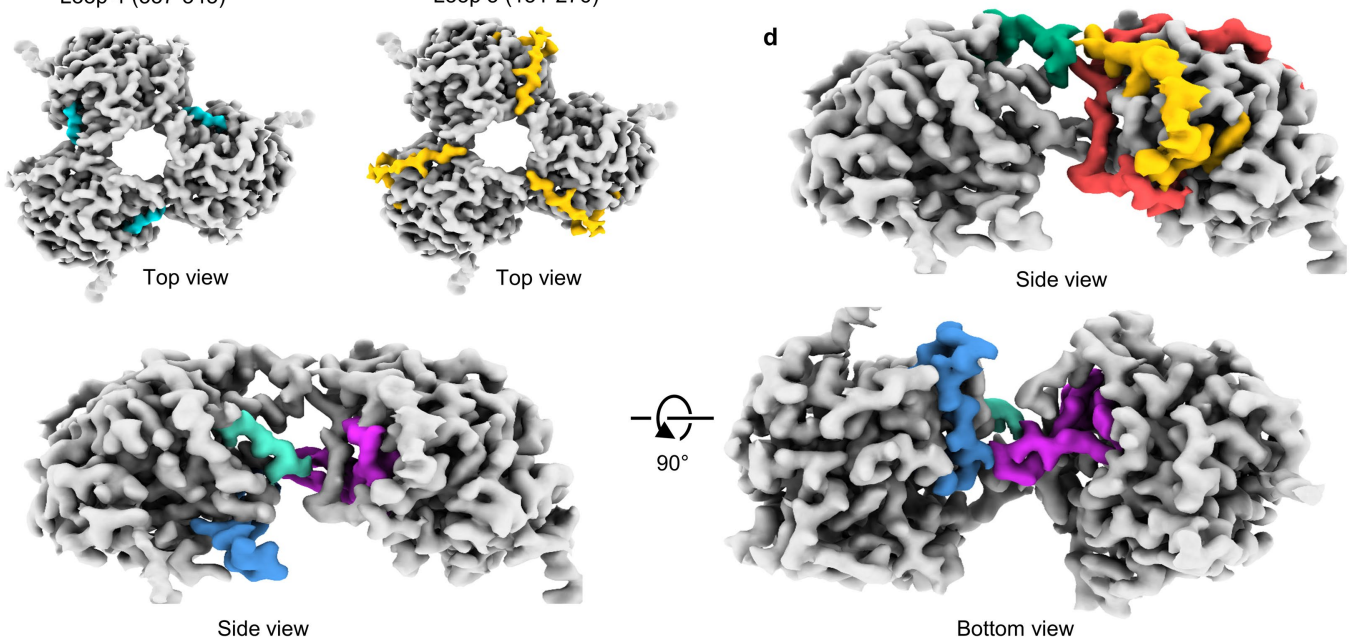

Extended Data Fig. $7 \mid \mathrm{MycP}_{5}$ caps a periplasmic cavity with its active site directed towards the lumen. a, Side and top view of an intact ESX-5 $5_{\mathrm{mtb}}$ assembly with $\mathrm{MycP}_{5}$ coloured as in Fig. 1 and the rest of the components transparent. Insets, side and top views of the periplasmic assembly with $\mathrm{EccB}_{5}$ in white, the $\mathrm{MycP}_{5}$ density shown in transparent red and loop 5 of $\mathrm{MycP}_{5}$ depicted in solid red at a higher threshold, to highlight it capping the periplasmic pore. Loop 5 folds along the protease domain, towards the pore

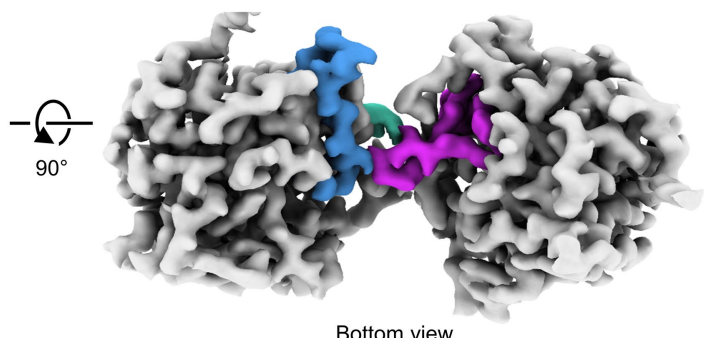

formed by the $\mathrm{MycP}_{5}$ trimer. At higher thresholds, loop 5 caps this pore. b. Top and side view of a dimer of $\mathrm{EccD}_{5}$ barrels, of which one barrel (left) binds via inner $\mathrm{EccD}_{5}$ to the $\mathrm{MycP}_{5}$ TMH.c, Top or bottom view of $\mathrm{MycP}_{5}$ trimers depicted in grey with the loops that are involved in $\mathrm{MycP}_{5}-\mathrm{MycP}_{5}$ interactions depicted in different colours. $\mathbf{d}$, Side and bottom views showing the $\mathrm{MycP}_{5}-$ $\mathrm{MycP}_{5}$ interactions mediated by the same domains depicted in the same colours as in $\mathbf{e}$. 

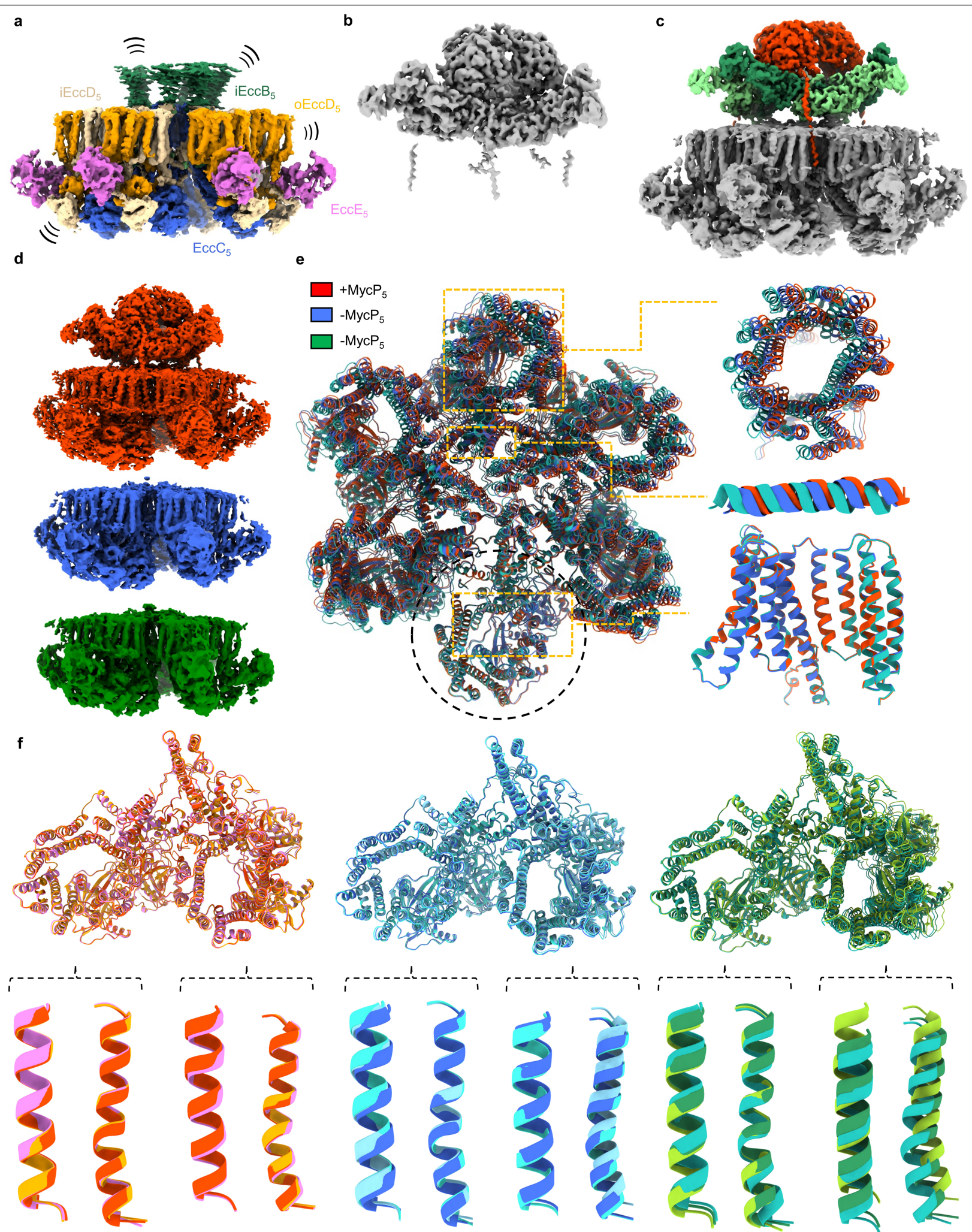

$\begin{array}{cccc}\mathrm{EccC}_{5} & \mathrm{EccD}_{5} & \mathrm{EccC}_{5} & \mathrm{EccD}_{5} \\ 227-245 & 437-461 & 227-245 & 437-461\end{array}$

$\underset{227-245}{\mathrm{EccC}_{5}}$

$\begin{array}{cc}\mathrm{EccD}_{5} & \mathrm{EccC}_{5} \\ 437-461 & 227-245\end{array}$

$\underset{437-461}{E c c D}$

$\underset{227-245}{\mathrm{EccC}_{5}}$

$\mathrm{EccD}_{5}$

$\mathrm{EccC}_{5}$

$\mathrm{EccD}_{5}$

Extended Data Fig. 8 |See next page for caption. 
Extended Data Fig. $8 \mid \mathrm{MycP}_{5}$ drives hexamerization of periplasmic EccB and complex stability. a, Cryo-EM density map of a $\mathrm{MycP}_{5}$-free ESX-5 $5_{\text {mtb }}$ membrane complex, zoned and coloured as in Fig. 1. In the absence of $\mathrm{MycP}_{5}$, the periplasmic domains of $\mathrm{EccB}_{5}$ display high flexibility. The rest of the membrane complex displays increased heterogeneity when compared to the $\mathrm{MycP}_{5}$-bound map. $\mathbf{b}$, Map of difference created by subtracting the $\mathrm{MycP}_{5}$-free map from the $\mathrm{MycP}_{5}$-bound map.c, Overlay of $\mathbf{a}$ and b. d, $\mathrm{MyCP}_{5}$-bound map in red and the two $\mathrm{MycP}_{5}$-free maps in blue and green.e, A model of the $\mathrm{MycP}_{5}{ }^{-}$ bound map, in which $\mathrm{MycP}_{5}$ and residues 84-504 of $\mathrm{EccB}_{5}$ were removed, was fitted into the models of the two $\mathrm{MycP}_{5}$-free maps, as described in Methods. Models were aligned at one $\mathrm{EccD}_{5}$ barrel (dark dotted circle), revealing substantial variations and shifts between the three maps. Top inset shows that there is consistent variation between all three maps at the membrane level $\left(\mathrm{EccD}_{5}\right.$ barrel). Middle inset shows variations between maps at cytosolic level $\left(\mathrm{EccB}_{5} \mathrm{~N}\right.$-terminal helix, residues 20-38). Bottom inset highlights inner $\mathrm{EccD}_{5}$ from the $\mathrm{EccD}_{5}$ barrel that was used for the alignment, showing that overall protomer structure does not change in the absence of $\mathrm{MycP}_{5}$. f, Dimers from every individual map, colour-coded the same as in d (different shades), were extracted and aligned to each other on one $\mathrm{EccD}_{5}$ barrel (left) as in e. Insets from these alignments, derived from both protomers, show that all three dimers of the $\mathrm{MycP}_{5}$-bound map show little to no variation, whereas the two $\mathrm{MycP}_{5}$-free maps show a higher degree of heterogeneity between dimers. 
a

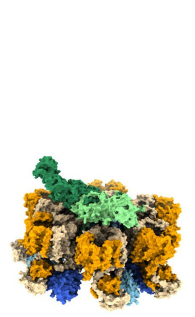

+3oxs

b
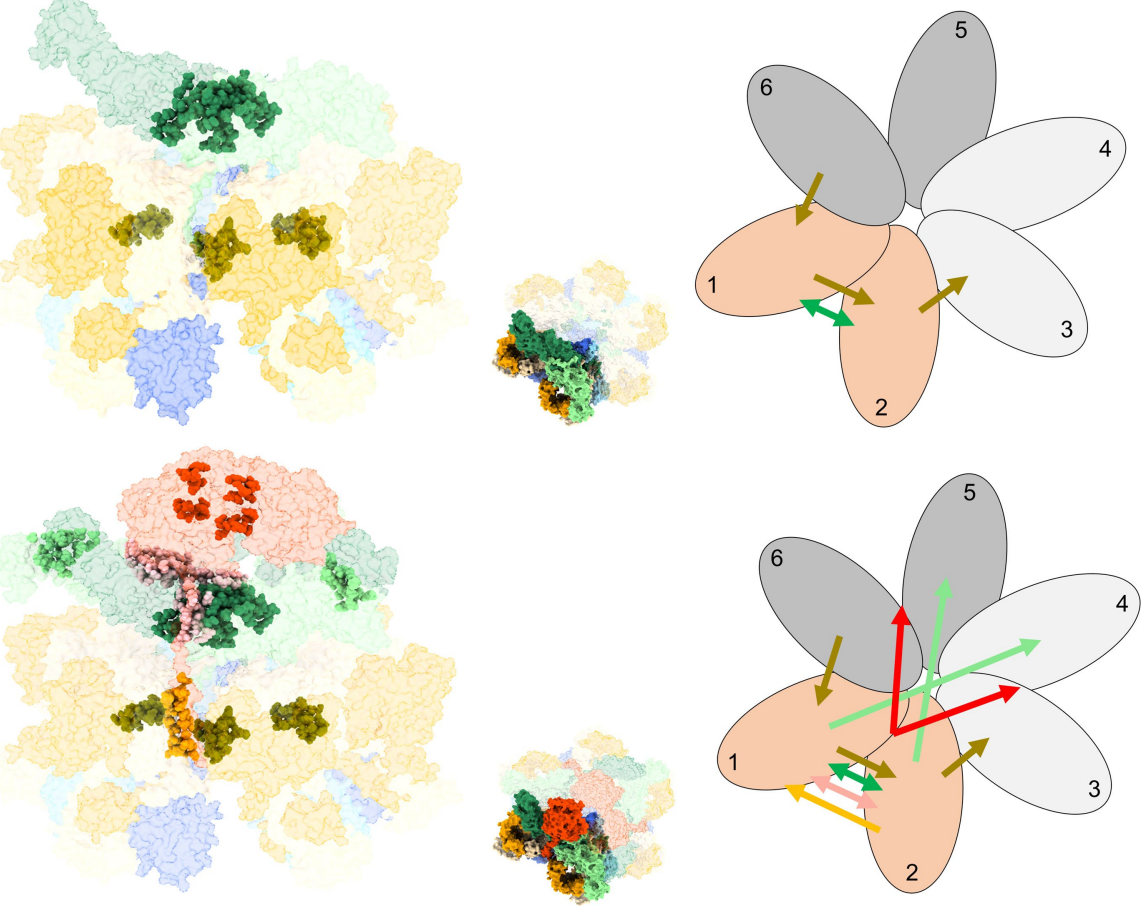

Intra-dimer contacts - $\mathrm{iEccB}_{5}-\mathrm{oEccB} \mathrm{iEccB}_{5}-\mathrm{MycP}_{5}-\mathrm{o} \mathrm{EccB}_{5} \mathrm{iEccB} \mathrm{B}_{5}-\mathrm{MycP}_{5}-\mathrm{iEccD} \mathrm{D}_{5}$ Inter-dimer contacts - $\mathrm{MycP}_{5}-\mathrm{MycP}_{5} \mathrm{i} \mathrm{EccB}_{5}-\mathrm{o} \mathrm{EccB}_{5} \mathrm{EccB}_{5}-\mathrm{iEccD} \mathrm{E}_{5}$

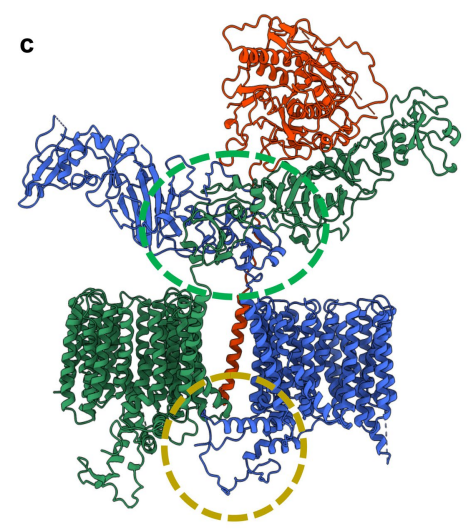

d
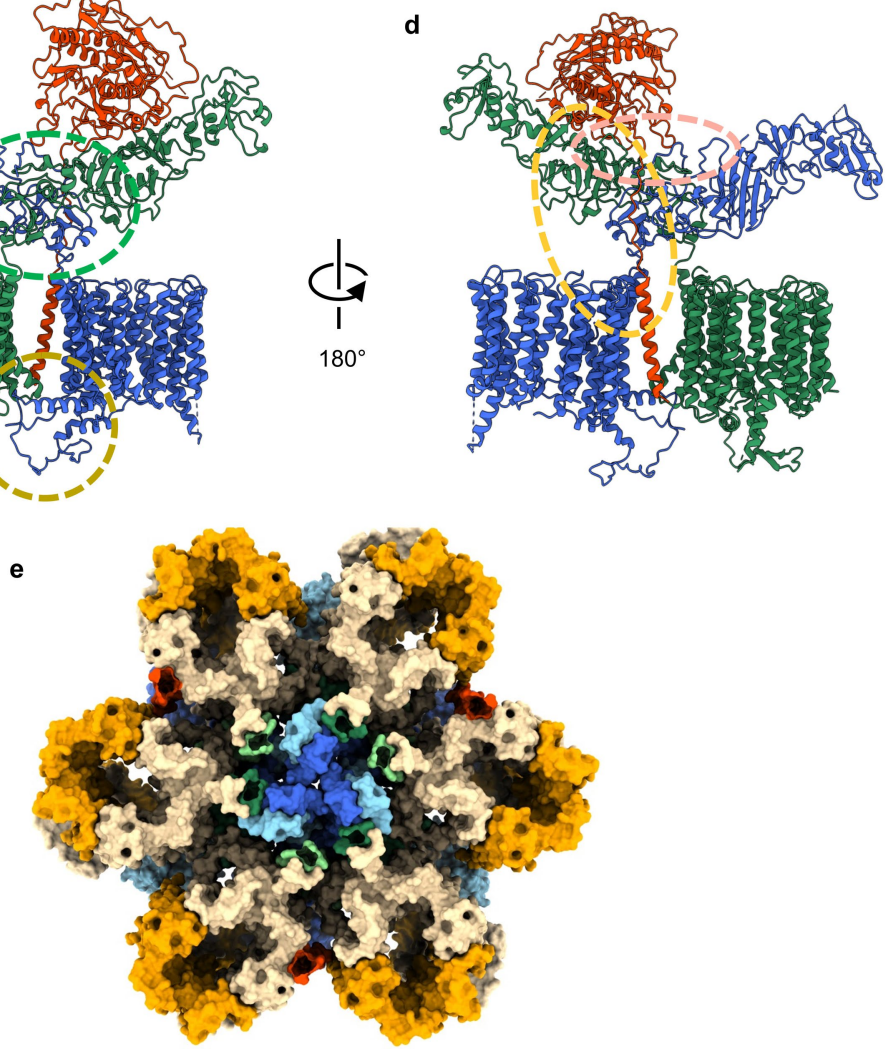

Extended Data Fig. $9 \mid$ See next page for caption. 
Extended Data Fig. $9 \mid \mathrm{MycP}_{5}$ creates more interaction points between protomers and dimers. a, Transparent surface model of a $\mathrm{MycP}_{5}$-free map with one $\mathrm{EccB}_{5}$ dimer at the periplasmic side, highlighting the interfaces of the protomers from a dimer. In the absence of $\mathrm{MycP}_{5}$, the two protomers from within one dimer exhibit two interactions: an $\mathrm{EccB}_{5}-\mathrm{EccB}_{5}$ interaction between their periplasmic domains, and a cytosolic one between $\mathrm{EccB}_{5}$-inner $\mathrm{EccD}_{5}$. The dimer further contacts the two immediate protomers of adjacent dimers through the mentioned $\mathrm{EccB}_{5}-\mathrm{EccD}_{5}$ cytosolic interaction. b. Transparent surface model of the $\mathrm{MycP}_{5}$-bound map, highlighting the interface of protomers from a dimer. On top of the mentioned contacts, in the presence of $\mathrm{MycP}_{5}$, protomers from a dimer interact with each other at the periplasmic side through inner $\mathrm{EccB}_{5}-\mathrm{MycP}_{5}-$ outer $\mathrm{EccB}_{5}$, while $\mathrm{MycP}_{5}$ further anchors the periplasmic assembly to the stable $\mathrm{EccD}_{5}$ raft through $\mathrm{EccB}_{5}-\mathrm{MycP}_{5}$-inner
$\mathrm{EccD}_{5} . \mathrm{MycP}_{5}$ also guides dimer-dimer interactions. By stabilizing the three $\mathrm{EccB}_{5}$ dimers in the triangle assembly, $\mathrm{MycP}_{5}$ promotes inner $\mathrm{EccB}_{5}$-outer $\mathrm{EccB}_{5}$ interactions between opposing protomers from adjacent dimers. Additionally, $\mathrm{MycP}_{5}$ promotes dimer-dimer contacts through $\mathrm{MycP}_{5}-\mathrm{MycP}_{5}$ interactions in the periplasm. Colour-coded legend applies to $\mathbf{a}, \mathbf{b} . \mathbf{c}, \mathbf{d}$, Inside (c) and outside (d) view of a dimer containing $\mathrm{EccB}_{5}, \mathrm{MycP}_{5}$ and the $\mathrm{TMHs}_{\mathrm{H}}$ of $\mathrm{EccD}_{5}$. For purposes of clarity, one $\mathrm{EccD}_{5}-\mathrm{EccB}_{5}$ protomer is coloured in blue, and the second protomer is in green and the $\mathrm{MycP}_{5}$ in red. Interactions between protomers of a dimer are highlighted and colour-coded as in a, b.e, Top view of a surface model missing the periplasmic domains of $\mathrm{EccB}_{5}$ and $\mathrm{MycP}_{5}$. The planes of protomers in which $\mathrm{MycP}_{5}$ binds inner $\mathrm{EcCD}_{5}$ are tilted by about $5^{\circ}$ compared to the $\mathrm{MycP}_{5}$-unbound ones. 

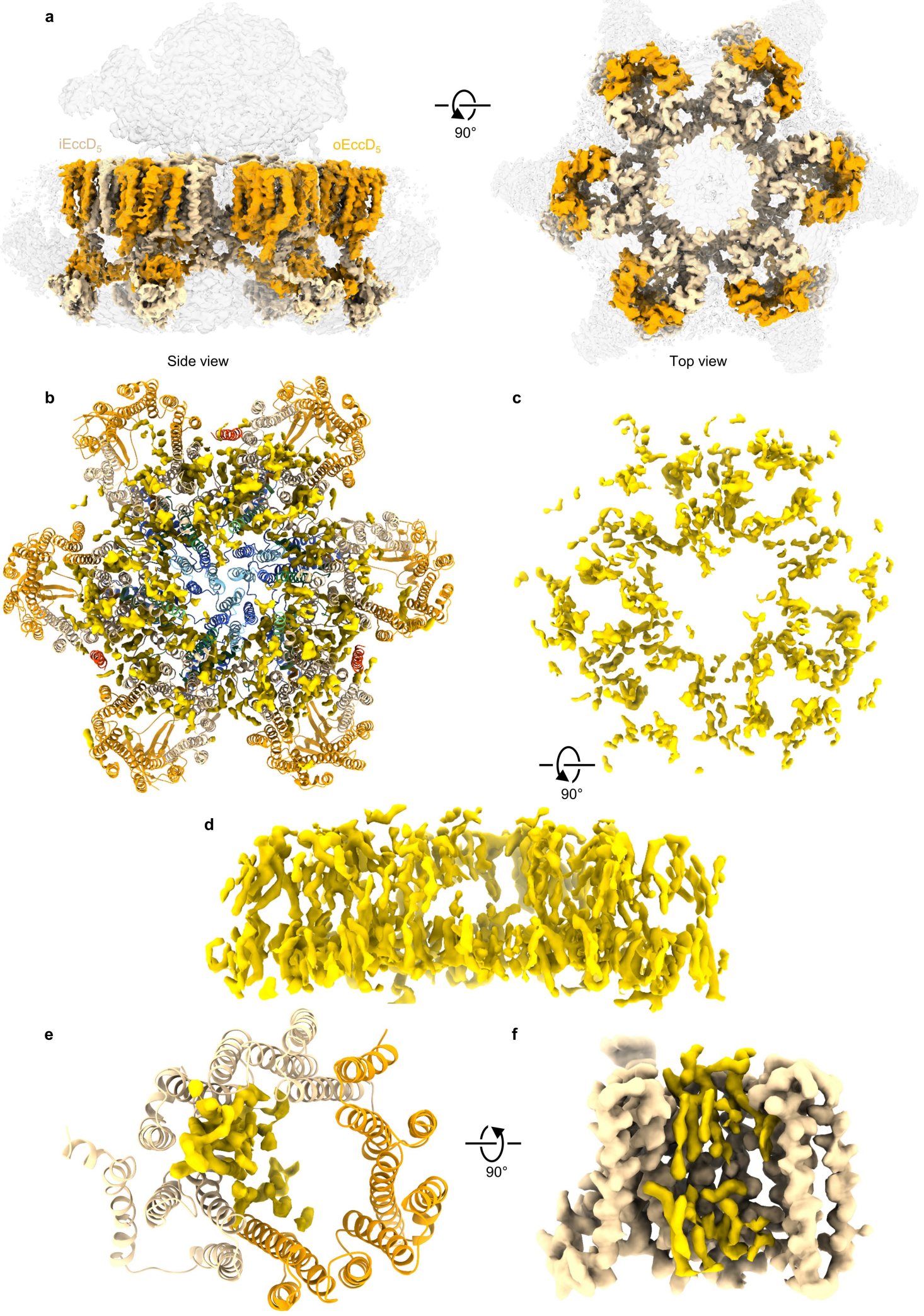

Extended Data Fig. 10 | Six lipid-filled EccD $_{5}$ barrels form a central raft. a, Side and top view of an intact ESX-5 $5_{\mathrm{mbb}}$ assembly, in which inner $\mathrm{EccD}_{5}$ and outer $\mathrm{EcCD}_{5}$ are coloured as in Fig. 1 and the rest of the components are transparent. b. Top view of the membrane region of the ESX-5 ${ }_{\mathrm{mtb}}$ model overlaid with observed lipids, coloured in bright yellow. c, Same view as b, but showing

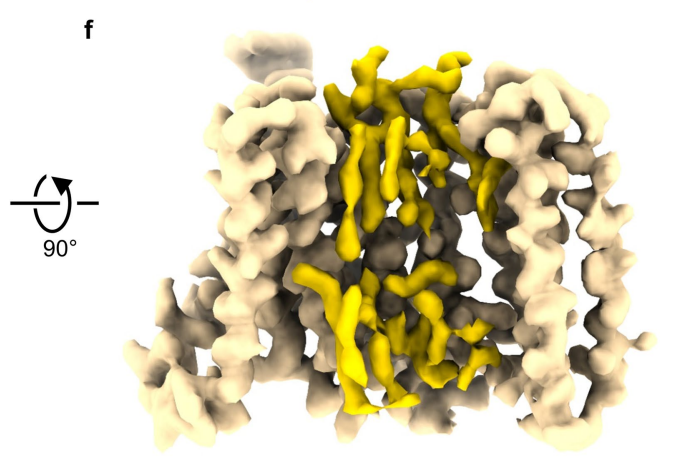

c
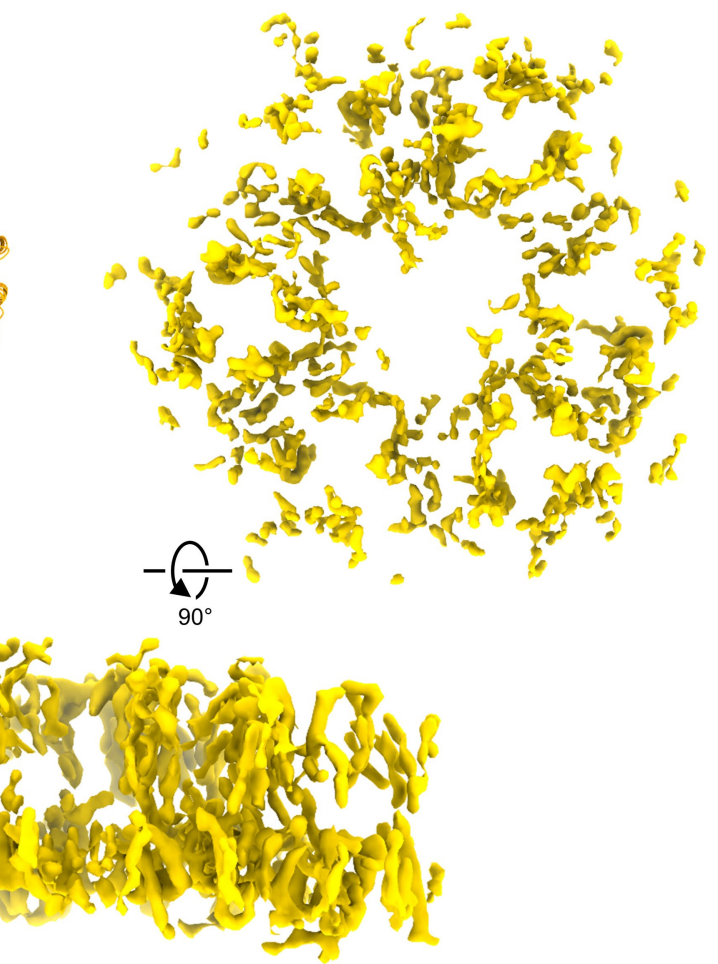

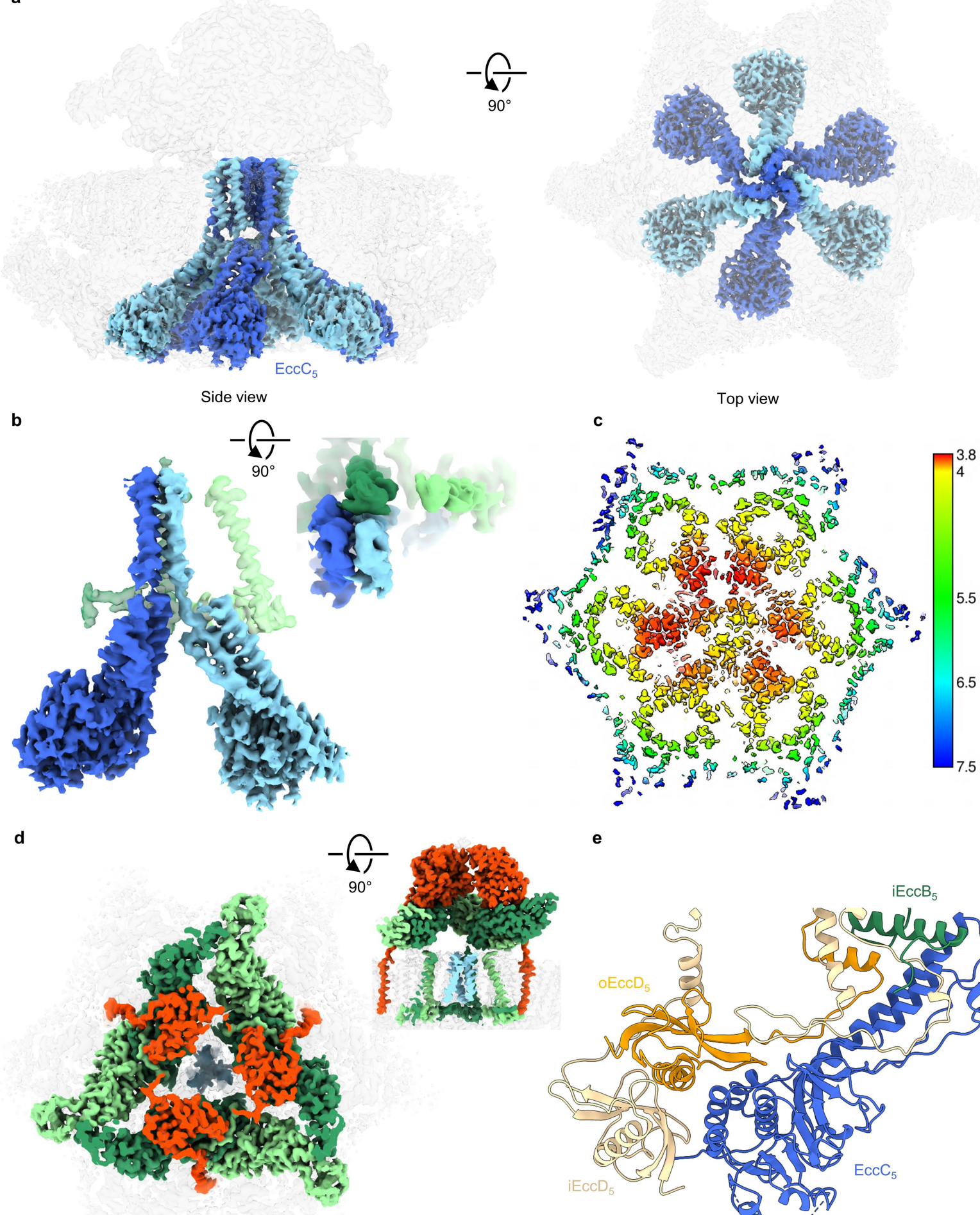

e

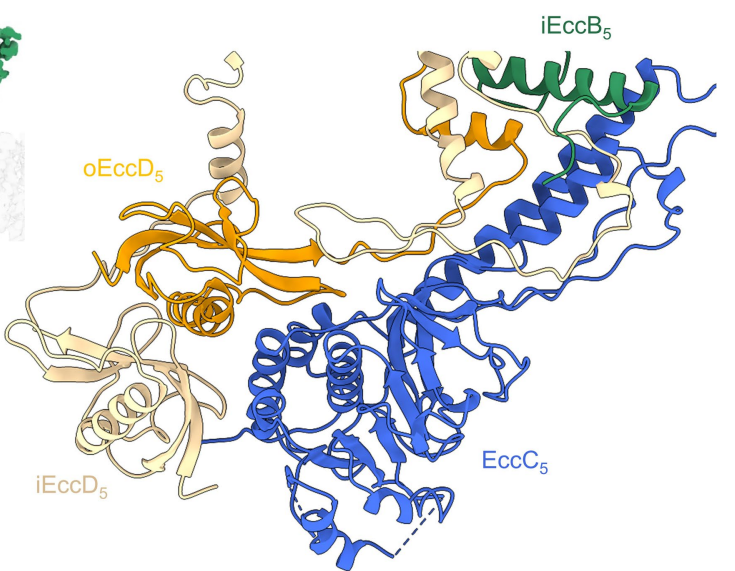

Extended Data Fig. 11 | Three four-TMH-bundles of $\mathrm{EccC}_{5}$ gate a central pore. a, Side and top view of an intact $\mathrm{ESX}-5_{\mathrm{mtb}}$ assembly, in which $\mathrm{EccC}_{5}$ is coloured in alternating light and dark blue and the rest of the components are transparent. b, Extracted dimeric $\mathrm{EccC}_{5}$ and the TMHs and $\mathrm{N}$ termini of $\mathrm{EccB}_{5}$ from the same dimer. The $90^{\circ}$ inset rotation shows that the TMH of outer $\mathrm{EccB}_{5}$ is not contacted by the TMHs of $\mathrm{EccC}_{5}$. c, Top membrane cross-section through a local-resolution map of a $\mathrm{C} 1$ full-complex reconstruction, displaying decreased resolution of the central space occupied by the $\mathrm{TMHs}_{\mathrm{M}} \mathrm{EccC}_{5}$, compared to the surrounding $\mathrm{EccB}_{5}$ basket and $\mathrm{TMH}_{\mathrm{H}}$ of inner $\mathrm{EccD}_{5}$. d, Top view of the full membrane complex with the $\mathrm{EccC}_{5} \mathrm{TMH}$ pyramid in light blue and the periplasmic $\mathrm{EccB}_{5}-\mathrm{MycP}_{5}$ in the same colours as in Fig. 1. The $\mathrm{EccC}_{5}$ $\mathrm{TMH}$ pyramid aligns with the periplasmic cavity and the $\mathrm{MycP}_{5}$-formed pore. $\mathrm{MycP}_{5}$ top part is partially sectioned, for clarity. Inset showing a $90^{\circ}$ rotation side cross-section of the same map.e, Ribbon model highlighting the structural features of the cytosolic bridge. 


\section{Article}

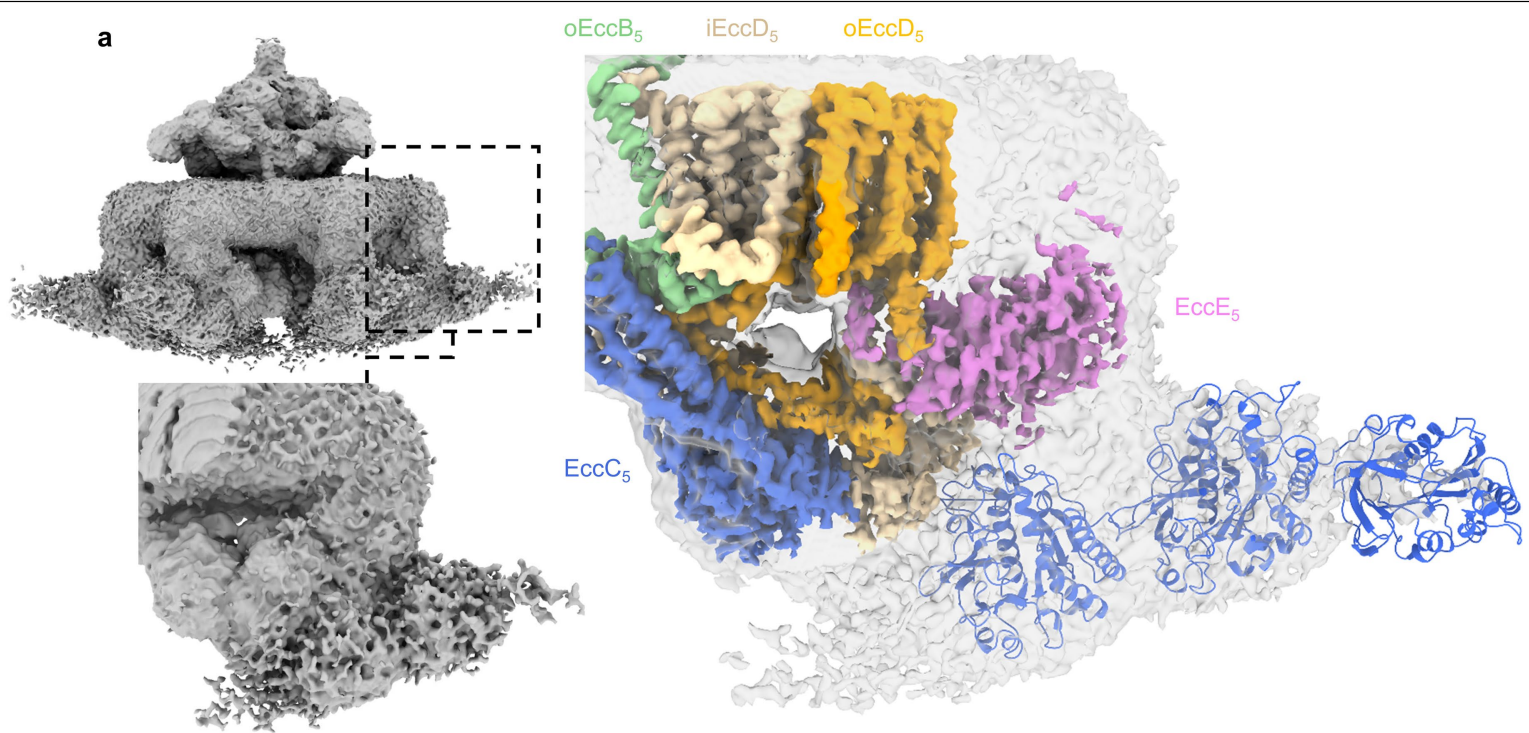

b
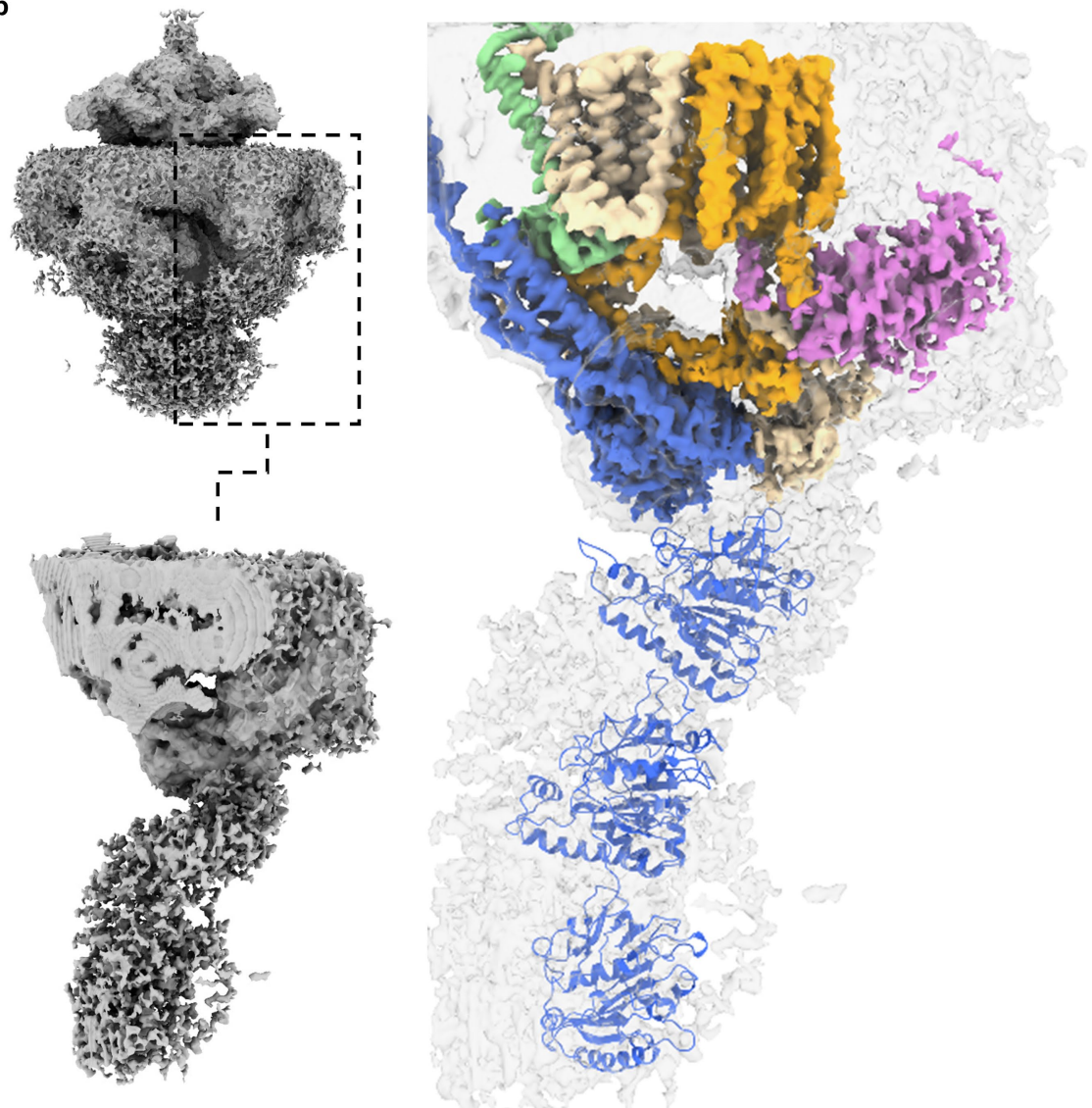

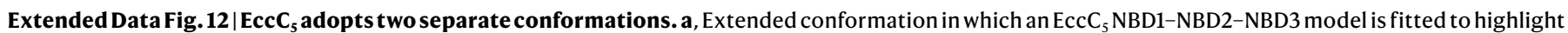
the overall position of these domains with respect to the rest of the membrane complex. b, As in a, but for the contracted conformation. 


\section{Reporting Summary}

Nature Research wishes to improve the reproducibility of the work that we publish. This form provides structure for consistency and transparency in reporting. For further information on Nature Research policies, see our Editorial Policies and the Editorial Policy Checklist.

\section{Statistics}

For all statistical analyses, confirm that the following items are present in the figure legend, table legend, main text, or Methods section.

n/a Confirmed

$\square \bigotimes$ The exact sample size $(n)$ for each experimental group/condition, given as a discrete number and unit of measurement

\ $\square$ A statement on whether measurements were taken from distinct samples or whether the same sample was measured repeatedly

$\square$ The statistical test(s) used AND whether they are one- or two-sided

Х $\square$ Only common tests should be described solely by name; describe more complex techniques in the Methods section.

Х $\square$ A description of all covariates tested

Х $\square$ A description of any assumptions or corrections, such as tests of normality and adjustment for multiple comparisons

$\square$ A full description of the statistical parameters including central tendency (e.g. means) or other basic estimates (e.g. regression coefficient)

$\triangle \square$ AND variation (e.g. standard deviation) or associated estimates of uncertainty (e.g. confidence intervals)

$\bigotimes \square \begin{aligned} & \text { For null hypothesis testing, the test statistic (e.g. } F, t, r \text { ) with confidence intervals, effect sizes, degrees of freedom and } P \text { value noted } \\ & \text { Give } P \text { values as exact values whenever suitable. }\end{aligned}$

\ $\square$ For Bayesian analysis, information on the choice of priors and Markov chain Monte Carlo settings

Х $\square$ For hierarchical and complex designs, identification of the appropriate level for tests and full reporting of outcomes

Х $\square$ Estimates of effect sizes (e.g. Cohen's $d$, Pearson's $r$ ), indicating how they were calculated

Our web collection on statistics for biologists contains articles on many of the points above.

\section{Software and code}

Policy information about availability of computer code

Data collection CryoEM: Thermo Fisher EPU V1.11 and V2.4; Negative stain EM: Thermo Fisher TIA V4.1.5; Western blotting: ChemoStar Touch (Intas Science Imaging Instruments GmbH, v. 0.5.65).

Data analysis MotionCor2 v.1.2.1 and v.1.3, CTFfind v.4.1.13, crYOLO v.1.4, Relion 3.1-beta, Chimera v.1.13.1 and 1.14, ChimeraX v.1 and v.1.1, Pymol v.2.40, Phyre2 (unversioned), Phenix v.1.18.2, Density modification (unversioned) and EMRinger (unversioned) both within the Phenix suite, DeepEMhancer (unversioned), ISOLDE v.1.0b5, MolProbity (unversioned), Phenix.real_space_refine v.1.18-6831, PISA (unversioned).

For manuscripts utilizing custom algorithms or software that are central to the research but not yet described in published literature, software must be made available to editors and reviewers. We strongly encourage code deposition in a community repository (e.g. GitHub). See the Nature Research guidelines for submitting code \& software for further information.

\section{Data}

Policy information about availability of data

All manuscripts must include a data availability statement. This statement should provide the following information, where applicable:

- Accession codes, unique identifiers, or web links for publicly available datasets

- A list of figures that have associated raw data

- A description of any restrictions on data availability

Cryo-EM maps have been deposited in the Electron Microscopy Database under accession codes EMD-12514 (full complex in C1), EMD-12517 (full complex in C3), EMD-12518 (periplasmic map in C1), EMD-12519 (periplasmic map in C3), EMD-12520 (cytosolic bridge), EMD-12521 (MycP5-free map 1), EMD-12522 (MycP5free map 2), EMD-12523 (EccC5 extended state) and EMD-12525 (EccC5 contracted state). The composite model settled in the C1 and C3 full maps, periplasm in C1, cytosolic bridge, MycP5-free map 1 and MycP5-free map 2 have been deposited in the Protein Data Bank under PDB accession codes 7NP7, 7NPR, 7NPS, 7NPT, 7NPU and 7NPV respectively. All other data is available from the corresponding author upon reasonable request. 


\section{Field-specific reporting}

Please select the one below that is the best fit for your research. If you are not sure, read the appropriate sections before making your selection.
$\mathrm{X}$ Life sciences
$\square$ Behavioural \& social sciences
Ecological, evolutionary \& environmental sciences

For a reference copy of the document with all sections, see nature.com/documents/nr-reporting-summary-flat.pdf

\section{Life sciences study design}

\begin{tabular}{|c|c|}
\hline Sample size & The size of the final particle set was determined by the ability to reach resolutions better than $4 \AA$ in $3 D$ reconstructions. \\
\hline Data exclusions & $\begin{array}{l}\text { Data was excluded during cryo-EM data processing by removing 2D and 3D classes that did not posses high-resolution features, a } \\
\text { standard method for cryo-EM high resolution structural determination. }\end{array}$ \\
\hline Replication & $\begin{array}{l}\text { All experiments have been successfully replicated. Solubilized membranes have been analyzed with BN-PAGE and EccB5 immunostaining } \\
\text { three times (ED Fig. 1B). Purifications +/- nucleotides (ED Fig. 1f, g) was replicated three times. Purification without nucleotides with EM } \\
\text { assessment has been performed three times (ED Fig. } 1 c, d, e) \text { and purification with nucleotides together with cryo-EM data collection has }\end{array}$ \\
\hline Randomization & $\begin{array}{l}\text { been performed two times (ED Fig. } 1 \mathrm{~h}, \mathrm{i}, \mathrm{j} \text { ). } \\
\text { Particles/images were randomly partitioned for resolution and quality assesment. }\end{array}$ \\
\hline linding & nding during data collection and analysis is not a commonly applied procedure in cryo-EM. \\
\hline
\end{tabular}

\section{Behavioural \& social sciences study design}

All studies must disclose on these points even when the disclosure is negative.

Study description Not relevant to this study
Research sample Not relevant to this study
Sampling strategy Not relevant to this study
Data collection
Timing Not revant to this study
Data exclusions Not revant to this study
Non-participation Not revant to this study
Randomization Not relevant to this study

\section{Ecological, evolutionary \& environmental sciences study design}

All studies must disclose on these points even when the disclosure is negative.
Study description
Not relevant to this study
Research sample
Not relevant to this study
Sampling strategy
Not relevant to this study
Data collection
Not relevant to this study
Timing and spatial scale
Not relevant to this study
Data exclusions
Not relevant to this study
Reproducibility
Not relevant to this study
Randomization
Not relevant to this study 
Did the study involve field work? $\square$ Yes $\square$ No

\section{Reporting for specific materials, systems and methods}

We require information from authors about some types of materials, experimental systems and methods used in many studies. Here, indicate whether each material, system or method listed is relevant to your study. If you are not sure if a list item applies to your research, read the appropriate section before selecting a response.

Materials \& experimental systems Methods

n/a $\quad$ Involved in the study

$\square$ Antibodies

$\mathrm{n} / \mathrm{a}$ Involved in the study

Х Eukaryotic cell lines

\ $\square$ Palaeontology and archaeology

Х $\square$ ChIP-seq

\ $\square$ Animals and other organisms

Х Flow cytometry

\ $\square$ Human research participants

$\bigotimes \square$ Clinical data

$\bigotimes \mid \square$ Dual use research of concern

\section{Antibodies}

Antibodies used

Validation anti EccB5 antibody

The anti EccB5 antibody was raised against the synthetic peptide CLPMDMSPAELVVPK by Innovagen (Lund, Sweden) and has been validated in doi:10.1111/j.1365-2958.2012.08206.x. It was used as a 1:5000 dilution. 